\title{
Orthogonal Systems with a Skew-Symmetric Differentiation Matrix
}

\author{
Arieh Iserles ${ }^{1} \cdot$ Marcus Webb ${ }^{2}$
}

Received: 17 April 2018 / Revised: 4 November 2018 / Accepted: 6 November 2018 /

Published online: 10 October 2019

(c) The Author(s) 2019

\begin{abstract}
In this paper, we explore orthogonal systems in $\mathrm{L}_{2}(\mathbb{R})$ which give rise to a real skewsymmetric, tridiagonal, irreducible differentiation matrix. Such systems are important since they are stable by design and, if necessary, preserve Euclidean energy for a variety of time-dependent partial differential equations. We prove that there is a one-to-one correspondence between such an orthonormal system $\left\{\varphi_{n}\right\}_{n \in \mathbb{Z}_{+}}$and a sequence of polynomials $\left\{p_{n}\right\}_{n \in \mathbb{Z}_{+}}$orthonormal with respect to a symmetric probability measure $\mathrm{d} \mu(\xi)=w(\xi) \mathrm{d} \xi$. If $\mathrm{d} \mu$ is supported by the real line, this system is dense in $\mathrm{L}_{2}(\mathbb{R})$; otherwise, it is dense in a Paley-Wiener space of band-limited functions. The path leading from $\mathrm{d} \mu$ to $\left\{\varphi_{n}\right\}_{n \in \mathbb{Z}_{+}}$is constructive, and we provide detailed algorithms to this end. We also prove that the only such orthogonal system consisting of a polynomial sequence multiplied by a weight function is the Hermite functions. The paper is accompanied by a number of examples illustrating our argument.
\end{abstract}

Keywords Differentiation matrix · Special functions · Orthogonal polynomials · Energy preserving numerical methods

Mathematics Subject Classification 42C05 - 65D25 - 42C30 - 65M12 - 65M70

Communicated by Petrushev, Pencho.

$\bowtie$ Marcus Webb

marcus.webb@manchester.ac.uk

1 Department of Applied Mathematics and Theoretical Physics Centre for Mathematical Sciences,

University of Cambridge, Wilberforce Road, Cambridge CB4 1LE, UK

2 Department of Mathematics, University of Manchester, Alan Turing Building, Manchester M13 9PL, UK 


\section{The Rationale}

\subsection{Differentiation Matrices}

The broad theme underlying this paper is the important benefits accrued in the semidiscretisation of time-dependent partial differential equations once space derivatives are approximated in a skew-symmetric (or skew-Hermitian in the complex case) manner. It is instructive to commence with three examples where, for simplicity, we assume a single spatial variable: the diffusion equation

$$
\frac{\partial u}{\partial t}=\frac{\partial}{\partial x}\left[a(x) \frac{\partial u}{\partial x}\right], \quad x \in[-1,1], t \geq 0,
$$

where $a(x)>0, x \in[-1,1]$, given with an initial condition for $t=0$ and either periodic or zero Dirichlet boundary conditions at $x= \pm 1$, the nonlinear advection equation

$$
\frac{\partial u}{\partial t}=\frac{\partial u}{\partial x}+f(u), \quad x \in \mathbb{R}, t \geq 0,
$$

where $v f(v) \leq 0$ for all $v \in \mathbb{R}$, given with an $\mathrm{L}_{2}(\mathbb{R})$ initial condition at $t=0$, and the linear Schrödinger equation in the semiclassical regime,

$$
\mathrm{i} \varepsilon \frac{\partial u}{\partial t}=-\varepsilon^{2} \frac{\partial^{2} u}{\partial x^{2}}+V(x) u, \quad x \in[-1,1], t \geq 0,
$$

given with an initial condition at $t=0$ and periodic boundary conditions at $x= \pm 1$. Here $0<\varepsilon \ll 1$, while the interaction potential $V$ is real.

It is a trivial exercise to prove that the solutions of both (1.1) and (1.2) are nonincreasing in the usual $L_{2}$ norm, while the $L_{2}$ norm of (1.3) is conserved. This represents a critical structural feature of the three equations which should ideally be preserved under discretisation. Moreover, once the $\mathrm{L}_{2}$ norm is uniformly bounded under discretisation, the underlying method is stable.

We commence from (1.1) and assume that it is semidiscretised by, say, finite differences, a spectral method or spectral collocation. ${ }^{1}$ The outcome is a set of linear ODEs,

$$
\boldsymbol{u}^{\prime}=\mathcal{D} \mathcal{A D} \boldsymbol{u}, \quad t \geq 0, \quad \boldsymbol{u}(0)=\boldsymbol{u}_{0},
$$

where $\mathcal{A}$ is positive definite. The matrix $\mathcal{D}$ is the result of discretising the space derivative, and we call this the differentiation matrix. Therefore,

$$
\frac{1}{2} \frac{\mathrm{d}\|\boldsymbol{u}\|^{2}}{\mathrm{~d} t}=\boldsymbol{u}^{\top} \boldsymbol{u}^{\prime}=\boldsymbol{u}^{\top} \mathcal{D} \mathcal{A} \mathcal{D} \boldsymbol{u}=\left(\mathcal{D}^{\top} \boldsymbol{u}\right) \mathcal{A}(\mathcal{D} \boldsymbol{u})
$$

\footnotetext{
${ }_{1}^{1}$ Semidiscretisation with finite elements requires trivial amendments to our argument, but its main thrust remains valid.
}

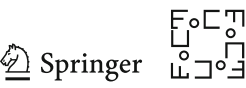


where $\|\cdot\|$ is the $\ell_{2}$ norm. Suppose now that the differentiation matrix $\mathcal{D}$ is skewsymmetric. Then $\mathcal{D}^{\top}=-\mathcal{D}$, and $\mathcal{A}$ being positive definite, it follows at once that $\mathrm{d}\|\boldsymbol{u}\|^{2} / \mathrm{d} t \leq 0$ : the numerical solution is dissipative (and, incidentally, stable!) Likewise, semidiscretising (1.2) with finite differences, we have

$$
\boldsymbol{u}^{\prime}=\mathcal{D} \boldsymbol{u}+\boldsymbol{f}(\boldsymbol{u}), \quad t \geq 0, \quad \boldsymbol{u}(0)=\boldsymbol{u}_{0},
$$

where $f_{m}(\boldsymbol{u})=f\left(u_{m}\right)$-we can again prove, identical to the above argument, that $\mathrm{d}\|\boldsymbol{u}\|^{2} / \mathrm{d} t \leq 0$ once $\mathcal{D}$ is skew-symmetric. Finally, semidiscretising the Schrödinger Eq. (1.3), we have

$$
\boldsymbol{u}^{\prime}=\mathrm{i} \varepsilon \mathcal{D}^{2} \boldsymbol{u}-\mathrm{i} \varepsilon^{-1} \mathcal{V} \boldsymbol{u}, \quad t \geq 0, \quad \boldsymbol{u}(0)=\boldsymbol{u}_{0},
$$

where the matrix $\mathcal{V}$ is real. Assuming again that $\mathcal{D}$ is skew-symmetric,

$$
\frac{1}{2} \frac{\mathrm{d}\|\boldsymbol{u}\|^{2}}{\mathrm{~d} t}=\operatorname{Re} \boldsymbol{u}^{*} \boldsymbol{u}^{\prime}=\operatorname{Re} \boldsymbol{u}^{*}\left(\mathrm{i} \varepsilon \mathcal{D}^{2} \boldsymbol{u}-\mathrm{i} \varepsilon^{-1} \mathcal{V} \boldsymbol{u}\right)=0
$$

because $\boldsymbol{u}^{\star} \mathcal{V} \boldsymbol{u}$ and $\boldsymbol{u}^{*} \mathcal{D}^{2} \boldsymbol{u}=\left(\mathcal{D}^{\top} \boldsymbol{u}\right)^{*}(\mathcal{D} \boldsymbol{u})=-\|\mathcal{D} \boldsymbol{u}\|^{2}$ are both real. Therefore, the semidiscretisation is conservative, mimicking the behaviour of the original equation. We should perhaps add that, in this context, $|u(x, t)|^{2}$ is the probability of finding a particle at $x$ : preservation of the norm is not an optional extra but a basic requirement once we wish to get the physics right.

Skew-symmetric differentiation matrices have been already analysed in some length in the context of finite differences in Hairer and Iserles [10,11] and Iserles [12,13]. The simplest second-order finite difference discretisation of a derivative,

$$
\mathcal{D}=\frac{1}{2 \Delta x}\left[\begin{array}{ccccc}
0 & 1 & 0 & \cdots & 0 \\
-1 & 0 & \ddots & \ddots & \vdots \\
0 & \ddots & \ddots & \ddots & 0 \\
\vdots & \ddots & \ddots & 0 & 1 \\
0 & \cdots & 0 & -1 & 0
\end{array}\right]
$$

is skew-symmetric, but this is a false dawn: this is the highest order skew-symmetric finite difference differentiation matrix on uniform grid [12]. It is possible to construct higher-order skew-symmetric differentiation matrices on special grids, but this is far from easy and large orders become fairly complicated [10,11]. Arguably this complexity makes them less suitable for efficient computation.

\subsection{Two Spectral Examples}

In this paper, we explore a general mechanism to generate orthogonal systems with skew-symmetric differentiation matrices since such systems can be implemented in the context of spectral methods. We do now address the issue of time discretisation,

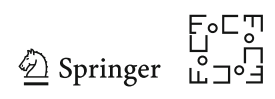


noting in passing that it might require a great deal of additional care, cf., for example, [1]. We commence with two examples. Firstly, consider the task of approximating a smooth periodic function on $[-\pi, \pi]$. The obvious choice in this setting is the Fourier basis, which we write in a real setting,

$$
\varphi_{0}(x) \equiv \frac{1}{(2 \pi)^{1 / 2}}, \quad \varphi_{2 n}(x)=\frac{\cos n x}{\pi^{1 / 2}}, \quad \varphi_{2 n+1}(x)=\frac{\sin n x}{\pi^{1 / 2}}, \quad n \in \mathbb{N}
$$

note that the basis is orthonormal. The differentiation matrix is

$$
\mathcal{D}=\left[\begin{array}{c|ccccccc}
0 & 0 & 0 & 0 & 0 & 0 & 0 & \cdots \\
\hline 0 & 0 & 1 & 0 & 0 & 0 & 0 & \cdots \\
0 & -1 & 0 & 0 & 0 & 0 & 0 & \cdots \\
0 & 0 & 0 & 0 & 2 & 0 & 0 & \cdots \\
0 & 0 & 0 & -2 & 0 & 0 & 0 & \cdots \\
0 & 0 & 0 & 0 & 0 & 0 & 3 & \cdots \\
0 & 0 & 0 & 0 & 0 & -3 & 0 & \cdots \\
\vdots & \vdots & \vdots & \vdots & \vdots & \vdots & \vdots & \ddots
\end{array}\right] .
$$

It is indeed skew-symmetric, as well as tridiagonal and reducible. Once we can use a Fourier basis, it must be an obvious first choice. The problem is, though, that periodic boundary conditions in a compact interval are something of an exception. Most partial differential equations of interest are either Cauchy problems defined on the entire Euclidean space or possess Dirichlet, Neumann or mixed boundary conditions in a compact domain. The focus of this paper is on Cauchy problems on the real line, and the motivation, which originates in (1.3) and its multivariate counterparts, is explained in greater detail in Iserles [13]. Briefly, periodic boundary conditions are useful for numerical simulation only in very short-time integration, leading to wrong behaviour once the solution (which originally has, to all intents and purposes, finite support and moves at finite speed) reaches the boundary. Modern computational challenges, not least in quantum control, call for a long-time solution of (1.3) and modern time-stepping methods (for example, those described in Bader et al. [1]) can indeed achieve the stability required to achieve this if there exists a discretisation yielding a skew-symmetric differentiation matrix. Hence, the challenge is to design and explore orthogonal systems on the real line with this property.

One example of such an orthogonal system, Hermite functions, is familiar in mathematical physics:

$$
\varphi_{n}(x)=\frac{(-1)^{n}}{\left(2^{n} n !\right)^{1 / 2} \pi^{1 / 4}} \mathrm{e}^{-x^{2} / 2} \mathrm{H}_{n}(x), \quad n \in \mathbb{Z}_{+}, x \in \mathbb{R},
$$

where $\mathrm{H}_{n}$ is the $n$th Hermite polynomial. It follows at once from standard theory of orthogonal polynomials that

$$
\int_{-\infty}^{\infty} \varphi_{m}(x) \varphi_{n}(x) \mathrm{d} x=\delta_{m, n}, \quad m, n \in \mathbb{Z}_{+} ;
$$


hence, $\left\{\varphi_{n}\right\}_{n \in \mathbb{Z}_{+}}$is indeed an orthonormal sequence, dense in $\mathrm{L}_{2}(\mathbb{R})$. It is well known (and can be confirmed easily using standard mixed recurrence relations for Hermite polynomials) that

$$
\varphi_{n}^{\prime}(x)=-\sqrt{\frac{n}{2}} \varphi_{n-1}(x)+\sqrt{\frac{n+1}{2}} \varphi_{n+1}(x), \quad n \in \mathbb{Z}_{+} ;
$$

therefore, the corresponding differentiation matrix is skew-symmetric - in addition, it is tridiagonal (making computations easier) and irreducible.

Hermite functions have many interesting features: they obey the Cramér inequality $\left|\varphi_{n}(x)\right| \leq 0.816049 \ldots, n \in \mathbb{Z}_{+}, x \in \mathbb{R}$, are eigenfunctions of the Fourier transform, obey a second-order linear ordinary differential equation and are related to Whittaker functions and parabolic cylinder functions. Insofar as this paper is concerned, (1.6) represents their most germane feature and a paradigm for more general systems of orthogonal functions in $\mathrm{L}_{2}(\mathbb{R})$.

\subsection{Plan of This Paper}

The purpose of this paper is to characterise all orthogonal systems in $\mathrm{L}_{2}(\mathbb{R})$ which possess a real skew-symmetric, tridiagonal, irreducible differentiation matrix.

An obvious approach is to consider different orthogonal systems $\left\{\varphi_{n}\right\}_{n \in \mathbb{Z}_{+}}$in $\mathrm{L}_{2}(\mathbb{R})$ and to check for each whether the differentiation matrix is of the right form - this hit-and-miss approach is unlikely to take us far. In Sect. 2, we adopt an alternative organising principle: we start from a countable sequence of functions $\left\{\varphi_{n}\right\}_{n \in \mathbb{Z}_{+}}$in $\mathrm{L}_{2}(\mathbb{R})$ which are hardwired to possess a skew-symmetric, tridiagonal, irreducible differentiation matrix and seek conditions for their orthogonality.

On the face of it, we have just replaced one hit-and-miss approach by another, yet there is crucial difference. In Sect. 3, we demonstrate that such a set of functions can be mapped by means of the Fourier transform, subject to rescaling, into a set $\left\{p_{n}\right\}_{n \in \mathbb{Z}_{+}}$of polynomials which are orthogonal with respect to some symmetric measure $\mathrm{d} \mu(x)=$ $w(x) \mathrm{d} x$. This argument can be reversed: given a real, symmetric measure $\mathrm{d} \mu$, we can construct an underlying set of orthonormal polynomials. These polynomials obey the familiar three-term recurrence relation from which we can recover the elements of $\mathcal{D}$. Finally, we recover the functions $\varphi_{n}$ by inverse Fourier transforming $\sqrt{w} p_{n}, n \in \mathbb{Z}_{+}$. It follows from the Parseval theorem that these functions form an orthonormal set. We prove that this set is dense in $L_{2}(\mathbb{R})$ if $d \mu$ is supported by $\mathbb{R}$; otherwise, it is dense in a Paley-Wiener space.

In Sect. 4, we describe practical algorithms for the evaluation of the $\varphi_{n} \mathrm{~s}$, in Sect. 5 we explore the structure of the functions $\left\{\varphi_{n}\right\}_{n \in \mathbb{Z}_{+}}$, while in Sect. 6 we present a number of examples. The paper concludes in Sect. 7 with a brief summary and discussion.

\section{The Main Paradigm}

The purpose of this paper is to investigate orthogonal systems $\Phi=\left\{\varphi_{n}\right\}_{n \in \mathbb{Z}_{+}}$of functions which are orthogonal on the real line with respect to the standard $L_{2}(\mathbb{R})$ inner 
product and such that their differentiation matrix $\mathcal{D}$ is skew-symmetric, tridiagonal and irreducible,

$$
\mathcal{D}=\left[\begin{array}{cccc}
0 & b_{0} & 0 & \cdots \\
-b_{0} & 0 & b_{1} & \ddots \\
0 & -b_{1} & \ddots & \ddots \\
\vdots & \ddots & \ddots & \ddots
\end{array}\right]
$$

where $b_{0}, b_{1}, \ldots$ are real, nonzero constants. In other words, we wish $\Phi$ to satisfy the skew-symmetric differentiation matrix relation

$$
\begin{aligned}
& \varphi_{0}^{\prime}(x)=b_{0} \varphi_{1}(x) \\
& \varphi_{n}^{\prime}(x)=-b_{n-1} \varphi_{n-1}(x)+b_{n} \varphi_{n+1}(x), \quad n \in \mathbb{N} .
\end{aligned}
$$

Given a seed function $\varphi_{0} \in \mathrm{C}^{\infty}(\mathbb{R})$, remaining functions can be defined uniquely by recursion from (2.1):

$$
\begin{aligned}
& n=0: \varphi_{1}(x)=\frac{1}{b_{0}} \varphi_{0}^{\prime}(x) \\
& n=1: \varphi_{2}(x)=\frac{1}{b_{1}}\left[\varphi_{1}^{\prime}(x)+b_{0} \varphi_{0}(x)\right]=\frac{1}{b_{0} b_{1}}\left[b_{0}^{2} \varphi_{0}(x)+\varphi_{0}^{\prime \prime}(x)\right] \\
& n=2: \varphi_{3}(x)=\frac{1}{b_{2}}\left[\varphi_{2}^{\prime}(x)+b_{1} \varphi_{1}(x)\right]=\frac{1}{b_{0} b_{1} b_{2}}\left[\left(b_{0}^{2}+b_{1}^{2}\right) \varphi_{0}^{\prime}(x)+\varphi_{0}^{\prime \prime \prime}(x)\right]
\end{aligned}
$$

and so on. In general, easy induction confirms that

$$
\varphi_{n}(x)=\frac{1}{b_{0} b_{1} \cdots b_{n-1}} \sum_{\ell=0}^{\lfloor n / 2\rfloor} \alpha_{n, \ell} \varphi_{0}^{(n-2 \ell)}(x), \quad n \in \mathbb{N}
$$

where

$$
\alpha_{n+1,0}=1, \quad \alpha_{n+1, \ell}=b_{n-1}^{2} \alpha_{n-1, \ell-1}+\alpha_{n, \ell}, \quad \ell=1, \ldots,\left\lfloor\frac{n}{2}\right\rfloor .
$$

The first thing to notice from this formula is that the $\varphi_{n}$ must indeed be a smooth (i.e. infinitely differentiable) function for all $n \in \mathbb{Z}_{+}$. Also note that, while cumbersome, this formula provides a practical method of generating examples.

Note, however, a major problem which provides the focus of this paper: the above procedure produces a set $\Phi$ which is guaranteed to satisfy (2.1), but a priori there is absolutely no reason why it should be orthogonal, whether with respect to a standard or any other inner product. Likewise, even if orthogonal, there is no a priori reason for $\Phi$ to be complete in the separable Hilbert space $\mathrm{L}_{2}(\mathbb{R})$.

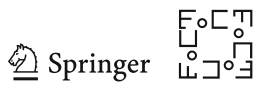


Example 1 (Hermite functions) Given the seed function and differentiation coefficients,

$$
\varphi_{0}(x)=\pi^{-\frac{1}{4}} \mathrm{e}^{-x^{2} / 2}, \quad b_{n}=\sqrt{\frac{n+1}{2}}, \quad n \in \mathbb{Z}_{+},
$$

the functions generated form the orthonormal Hermite function basis, which we have already encountered in Sect. 1.

Example 2 (Spherical Bessel functions) Given the seed function and differentiation coefficients

$$
\varphi_{0}(x)=\frac{1}{\sqrt{2}} \frac{\mathrm{J}_{\frac{1}{2}}(x)}{\sqrt{x}}=\pi^{-\frac{1}{2}} \mathrm{j}_{0}(x), \quad b_{n}=\frac{n+1}{\sqrt{(2 n+1)(2 n+3)}}, \quad n \in \mathbb{Z}_{+},
$$

the functions generated are

$$
\varphi_{n}(x)=\sqrt{\frac{n+\frac{1}{2}}{x}} \mathrm{~J}_{n+\frac{1}{2}}(x)=\sqrt{\frac{2 n+1}{\pi}} \mathrm{j}_{n}(x),
$$

which are the spherical Bessel functions. Note that while the Bessel functions $\mathrm{J}_{n+\frac{1}{2}}(z)$ have a branch cut in $(-\infty, 0]$, the spherical Bessel function $\mathrm{j}_{n}(z)$ is entire.

Example 3 (Quasi-Hermite) Suppose we use the Gaussian seed function and constant differentiation coefficients,

$$
\varphi_{0}(x)=\mathrm{e}^{-x^{2}}, \quad b_{n}=1, \quad n \in \mathbb{Z}_{+} .
$$

Then the generated function sequence is

$$
\begin{gathered}
\varphi_{2 n}(x)=\mathrm{e}^{-x^{2}} \sum_{\ell=0}^{n}\left(\begin{array}{c}
n+\ell \\
2 \ell
\end{array}\right) \mathrm{H}_{2 \ell}(x), \\
\varphi_{2 n+1}(x)=\mathrm{e}^{-x^{2}} \sum_{\ell=0}^{n}\left(\begin{array}{c}
n+\ell+1 \\
2 \ell+1
\end{array}\right) \mathrm{H}_{2 \ell+1}(x),
\end{gathered}
$$

where $\mathrm{H}_{k}(x)$ is the $k$ th Hermite polynomial. We do not prove this formula since, as will transpire in Sect. 5, such functions cannot be orthogonal, and hence are of little merit.

To recap, whatever the merits of (2.2) - and is certainly useful for generating the desired function sequence $\left\{\varphi_{n}\right\}_{n \in \mathbb{Z}_{+}}$-this construction does not make obvious what properties the sequence might have, such as orthogonality or its norm. Indeed, the Hermite functions and the spherical Bessel functions generating the above examples turn out to be orthonormal in $\mathrm{L}_{2}(\mathbb{R})$, but it is not obvious from this construction that would happen. It can be shown that the function sequence generated in Example 3 is 
not orthogonal with respect to any real-valued measure. Other types of constructions will be considered in Sects. 3 and 4, for which the orthogonality of the sequence can be more obviously predicted.

We commenced this section specifying a seed $\varphi_{0}$ and nonzero real coefficients $\left\{b_{n}\right\}_{n \in \mathbb{Z}_{+}}$. As becomes clear in the next section, once we seek orthogonality, the right procedure is more minimalistic: it is enough to choose a symmetric positive Borel measure $\mathrm{d} \mu$ on the real line, which determines both the seed and the coefficients in a unique manner while ensuring orthogonality and completeness in a certain Hilbert space.

\section{The Fourier Transform and Orthogonal Polynomials}

\subsection{There and Back Again}

We commence our journey from a given sequence of real-valued, square-integrable, smooth functions $\Phi=\left\{\varphi_{n}\right\}_{n \in \mathbb{Z}_{+}}$, which has a skew-symmetric differentiation matrix with nonzero real constants $\left\{b_{n}\right\}_{n \in \mathbb{Z}_{+}}$as in (2.1). For our departure, we require the unitary Fourier transform,

$$
\mathcal{F}[\varphi](\xi)=\frac{1}{\sqrt{2 \pi}} \int_{-\infty}^{\infty} \varphi(x) e^{-\mathrm{i} x \xi} \mathrm{d} x .
$$

As is well known, the Fourier transform and differentiation have the commutation relation

$$
\mathcal{F}\left[\varphi^{\prime}\right](\xi)=-\mathrm{i} \xi \mathcal{F}[\varphi](\xi) .
$$

This motivates our definition of a transformed sequence of functions, $\Psi=\left\{\psi_{n}\right\}_{n \in \mathbb{Z}_{+}}$, where

$$
\psi_{n}(\xi)=\mathrm{i}^{n} \mathcal{F}\left[\varphi_{n}\right](\xi)
$$

Each $\varphi_{n}$ has a well-defined Fourier transform, being square-integrable on the real line. From the equation for differentiation of a Fourier transform given above, we have for all $n \in \mathbb{Z}_{+}$,

$$
\xi \psi_{n}(\xi)=\mathrm{i}^{n} \xi \mathcal{F}\left[\varphi_{n}\right](\xi)=\mathrm{i}^{n+1}(-\mathrm{i} \xi) \mathcal{F}\left[\varphi_{n}\right](\xi)=\mathrm{i}^{n+1} \mathcal{F}\left[\varphi_{n}^{\prime}\right](\xi) .
$$

Next, using the skew-symmetric differentiation property of $\Phi$, we see that the transformed functions $\Psi$ satisfy

$$
\begin{aligned}
& \xi \psi_{0}(\xi)=b_{0} \mathrm{i} \mathcal{F}\left[\varphi_{1}\right](\xi)=b_{0} \psi_{1}(\xi) \\
& \xi \psi_{n}(\xi)=-b_{n-1} \mathrm{i}^{n+1} \mathcal{F}\left[\varphi_{n-1}\right](\xi)+b_{n} \mathrm{i}^{n+1} \mathcal{F}\left[\varphi_{n+1}\right](\xi)=b_{n-1} \psi_{n-1}(\xi)+b_{n} \psi_{n+1}(\xi)
\end{aligned}
$$

In other words, they satisfy a three-term recurrence relation! A simple consequence of this is $\psi_{n}(\xi)=p_{n}(\xi) \psi_{0}(\xi)$, where $P=\left\{p_{n}\right\}_{n \geq 0}$ is a sequence of polynomials

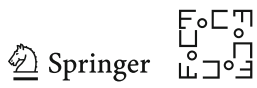


satisfying a three-term recurrence,

$$
\begin{aligned}
p_{0}(\xi) & =1, \quad p_{1}(\xi)=b_{0}^{-1} \xi \\
p_{n+1}(\xi) & =\frac{\xi}{b_{n}} p_{n}(\xi)-\frac{b_{n-1}}{b_{n}} p_{n-1}(\xi), \quad n \in \mathbb{N}
\end{aligned}
$$

We can now use the classical Favard theorem $[3,6]$ to deduce that these polynomials are orthogonal with respect to a real-valued, finite Borel measure.

Theorem 4 (Favard) Let $P=\left\{p_{n}\right\}_{n \geq 0}$ be a sequence of real polynomials such that $\operatorname{deg}\left(p_{n}\right)=n . P$ is an orthogonal system with respect to the inner product $\langle f, g\rangle_{\mu}=$ $\int \overline{f(\xi)} g(\xi) \mathrm{d} \mu(\xi)$ for some probability measure ${ }^{2} \mathrm{~d} \mu$ on the real line if and only if the polynomials satisfy the three-term recurrence,

$$
p_{n+1}(\xi)=\left(\alpha_{n}-\beta_{n} \xi\right) p_{n}(\xi)-\gamma_{n} p_{n-1}(\xi), \quad n \in \mathbb{Z}_{+},
$$

for some real sequences $\left\{\alpha_{n}\right\}_{n \in \mathbb{Z}_{+}},\left\{\beta_{n}\right\}_{n \in \mathbb{Z}_{+}},\left\{\gamma_{n}\right\}_{n \in \mathbb{Z}_{+}}$with $\gamma_{0}=0$ and $\gamma_{n} \beta_{n-1} / \beta_{n}>$ 0 for all $n \in \mathbb{N}$.

The polynomials from Eq. (3.3) fall under the umbrella of Favard's theorem, with $\alpha_{n}=0, \beta_{n}=-b_{n}^{-1}$ and $\gamma_{n}=b_{n-1} b_{n}^{-1}$, because

$$
\frac{\gamma_{n} \beta_{n-1}}{\beta_{n}}=\frac{\left(b_{n-1} b_{n}^{-1}\right)\left(-b_{n-1}^{-1}\right)}{-b_{n}^{-1}}=1>0 .
$$

We can now deduce even more about the polynomials, because there exist simple relationships between the polynomials, the coefficients of the three-term recurrence and the measure of orthogonality, as follows.

Lemma 5 Let $P$ be as in Favard's theorem above.

1. The polynomials are orthonormal if and only if $\gamma_{n} \beta_{n-1} / \beta_{n}=1$ for all $n \in \mathbb{N}$ and $\int p_{0}(\xi)^{2} \mathrm{~d} \mu(\xi)=1$.

2. The measure $\mathrm{d} \mu$ is symmetric [i.e. $\mathrm{d} \mu(-\xi)=\mathrm{d} \mu(\xi)$ ] if and only if $\alpha_{n}=0$ for all $n \in \mathbb{Z}_{+}$. In this case, $p_{n}(-\xi)=(-1)^{n} p_{n}(\xi)$ for all $n \in \mathbb{Z}_{+}$.

3. For each $n \geq 0$, the sign of $\beta_{n}$ is negative if and only if the signs of the leading coefficient of $p_{n}$ and $p_{n+1}$ are the same. Otherwise $\beta_{n}$ is positive.

Proof We sketch the proofs because they are elementary but not obvious. Multiplying the three-term recurrence by $p_{n+1}$, integrating, dropping the terms which are zero by orthogonality, and then applying the three-term recurrence to $\xi p_{n}(\xi)$ (and dropping more terms which are zero by orthogonality) show that

$$
\int p_{n}(\xi)^{2} \mathrm{~d} \mu(\xi)=\frac{\gamma_{n} \beta_{n-1}}{\beta_{n}} \int p_{n-1}(\xi)^{2} \mathrm{~d} \mu(\xi) .
$$

\footnotetext{
2 A probability measure is a scalar-valued Borel measure which is positive and has total mass equal to 1.
} 
This proves part 1 of this lemma. Part 2 of the lemma is proved in Theorem 4.3 of Chihara [3]. Part 3 follows from the fact that the leading coefficient of $p_{n+1}$ is equal to the leading coefficient of $-\beta_{n} \xi p_{n}(\xi)$, which equals the leading coefficient of $p_{n}$ times $-\beta_{n}$.

By Lemma 5 parts 1 and 2, the polynomials in Eq. (3.3) are always orthonormal with respect to a symmetric probability measure $\mathrm{d} \mu$ on the real line. Note that this is regardless of the signs and magnitudes of each $b_{n}$. In fact, by part 3 of Lemma 5 , the leading coefficients of these orthonormal polynomials will have signs which depend on the sign of each $b_{n}$.

Taking stock, we see that our journey has led us from the sequence of functions $\Phi=\left\{\varphi_{n}\right\}_{n \in \mathbb{Z}_{+}}$with a real, skew-symmetric, irreducible differentiation matrix, to the transformed functions $\Psi=\left\{\psi_{n}\right\}_{n \in \mathbb{Z}_{+}}$, which are of the form $\psi_{n}(\xi)=p_{n}(\xi) g(\xi)$, where $P=\left\{p_{n}\right\}_{n \in \mathbb{Z}_{+}}$are orthonormal polynomials with respect to a symmetric probability measure $\mathrm{d} \mu$ and $g(\xi)=\psi_{0}(\xi)=\mathcal{F}\left[\varphi_{0}\right](\xi)$. By Eq. (2.2), $\varphi_{0}$ is infinitely differentiable, which implies that $g(\xi) \rightarrow 0$ superalgebraically as $|\xi| \rightarrow \infty$. Since we assume that $\varphi_{0}$ is real-valued, then the functions $g=\mathcal{F}\left[\varphi_{0}\right]$ must have an even real part and an odd imaginary part (although in all specific cases considered in this paper, $g$ is real-valued and even). Furthermore, since $\varphi_{0}$ is square-integrable, so is $g$. In the current context, we refer to such functions as mollifiers.

Let us now embark on a return journey. Commencing with a symmetric probability measure $\mathrm{d} \mu$ on the real line, we let $P=\left\{p_{n}\right\}_{n \in \mathbb{Z}_{+}}$be a family of real orthonormal polynomials for the measure $\mathrm{d} \mu$, with $p_{0}=1$. Then by Favard's theorem and Lemma 5 , there exists a sequence of nonzero real numbers $\left\{b_{n}\right\}_{n \in \mathbb{Z}_{+}}$such that the three-term recurrence (3.3) holds. Also by Lemma 5, the sign of each $b_{n}$ depends on the changes in sign of the leading coefficients of the polynomials, which can be arbitrary.

Define the functions $\Phi=\left\{\varphi_{n}\right\}_{n \in \mathbb{Z}_{+}}$by,

$$
\varphi_{n}(x)=(-\mathrm{i})^{n} \mathcal{F}^{-1}\left[g \cdot p_{n}\right]
$$

where $g$ is a mollifier as discussed above. By Lemma 5 part 2, $p_{n}$ is has the same parity as a function as $n$ has as an integer. Since $g$ has even real part and odd imaginary part, this implies that $(-\mathrm{i})^{n} g \cdot p_{n}$ has even real part and odd imaginary part too. This implies that $\varphi_{n}$ is real-valued for all $n \in \mathbb{Z}_{+}$. Furthermore, since $g$ is square-integrable and decays superalgebraically to zero at infinity, this implies that $g \cdot p_{n}$ is square-integrable, and hence is $\varphi_{n}$ for all $n \in \mathbb{Z}_{+}$.

It is our intent that $\Phi$ has a skew-symmetric differentiation matrix. Indeed, using the equation for differentiation of a Fourier transform [Eq. (3.2)] and the three-term recurrence for $P$, we have

$$
\begin{aligned}
\varphi_{n}^{\prime} & =\mathcal{F}^{-1}\left[(-\mathrm{i} \xi) \mathcal{F}\left[\varphi_{n}\right](\xi)\right]=\mathcal{F}^{-1}\left[(-\mathrm{i})^{n+1} \xi g(\xi) p_{n}(\xi)\right] \\
& =b_{n-1}(-\mathrm{i})^{n+1} \mathcal{F}^{-1}\left[g \cdot p_{n-1}\right]+b_{n}(-\mathrm{i})^{n+1} \mathcal{F}^{-1}\left[g \cdot p_{n+1}\right] \\
& =-b_{n-1} \varphi_{n-1}+b_{n} \varphi_{n+1} .
\end{aligned}
$$


Similarly, $\varphi_{0}^{\prime}=b_{0} \varphi_{1}$. The return journey goes off without a hitch! The mollifier $g$ is also uniquely determined by the family $\Phi$ : we have $g=\mathcal{F}\left[\varphi_{0}\right]$, because $p_{0}=1$. This proves the following theorem.

Theorem 6 (Fourier characterisation for $\Phi$ ) The sequence $\Phi=\left\{\varphi_{n}\right\}_{n \in \mathbb{Z}_{+}}$of realvalued, square-integrable functions has a real, skew-symmetric, tridiagonal, irreducible differentiation matrix if and only if

$$
\varphi_{n}(x)=\frac{(-\mathrm{i})^{n}}{\sqrt{2 \pi}} \int_{-\infty}^{\infty} \mathrm{e}^{\mathrm{i} x \xi} p_{n}(\xi) g(\xi) \mathrm{d} \xi
$$

where $P=\left\{p_{n}\right\}_{n \in \mathbb{Z}_{+}}$is an orthonormal polynomial system on the real line with respect to a symmetric probability measure $\mathrm{d} \mu$ and $g$ is mollifier (as defined above). Furthermore, $P$ and $g$ are uniquely determined by $\Phi$ and $\left\{b_{n}\right\}_{n \in \mathbb{Z}_{+}}$.

Remark 7 Relaxing the conditions on what it means to be a mollifier yield further examples of sequences $\Phi$ with a real, skew-symmetric, tridiagonal, irreducible differentiation matrix, but which are not necessarily square-integrable or real-valued. Furthermore, relaxing the symmetry condition on the measure $\mathrm{d} \mu$ leads to skewHermitian differentiation matrices. We do not pursue these extensions in this paper.

\subsection{Orthogonal Bases}

It turns out that this characterisation using the Fourier transform lends itself well to the determination of orthogonality. Recall Parseval's theorem, namely that the unitary Fourier transform in (3.1) satisfies

$$
\int_{-\infty}^{\infty} \overline{\varphi(x)} \psi(x) \mathrm{d} x=\int_{-\infty}^{\infty} \overline{\mathcal{F}[\varphi](\xi)} \mathcal{F}[\psi](\xi) \mathrm{d} \xi,
$$

for all $\varphi, \psi \in \mathrm{L}_{2}(\mathbb{R})$.

Theorem 8 (Orthogonal bases) Let $\varphi_{n}=(-\mathrm{i})^{n} \mathcal{F}^{-1}\left[g \cdot p_{n}\right]$ for $n \in \mathbb{Z}_{+}$as in Theorem 6. Then $\Phi$ is orthogonal in $\mathrm{L}_{2}(\mathbb{R})$ if and only if $P$ is orthogonal with respect to the measure $|g(\xi)|^{2} \mathrm{~d} \xi$. Furthermore, whenever $\Phi$ is orthogonal, the functions $\varphi_{n} /\|g\|_{2}$ are orthonormal.

Proof By Parseval's theorem,

$$
\int_{-\infty}^{\infty} \overline{\varphi_{n}(x)} \varphi_{m}(x) \mathrm{d} x=(-\mathrm{i})^{m-n} \int_{-\infty}^{\infty} p_{n}(\xi) p_{m}(\xi)|g(\xi)|^{2} \mathrm{~d} \xi .
$$

Clearly $\Phi$ is orthogonal if and only if $P$ is orthogonal with respect to $|g(\xi)|^{2} \mathrm{~d} \xi$. For the final part of the theorem, recall the earlier discussion that $P$ is always orthonormal with respect to the probability measure $\mathrm{d} \mu(\xi) / \int p_{0}^{2} \mathrm{~d} \mu(\xi)$. Therefore, in the present case, $P$ is orthonormal with respect to $|g(\xi)|^{2} \mathrm{~d} \xi /\|g\|_{2}^{2}$. Therefore, $\left\|\varphi_{n}\right\|_{2}=\|g\|_{2}$ for all $n \in \mathbb{Z}_{+}$. This completes the proof. 
What Theorems 6 and 8 show is that there is a one-to-one correspondence between orthogonal systems $\Phi$ with real, skew-symmetric, irreducible differentiation matrices and sequences $P$ of orthonormal polynomials with respect to a symmetric probability measure of the form $\mathrm{d} \mu(\xi)=w(\xi) \mathrm{d} \xi$. (Note that we allow the leading coefficients of the orthonormal polynomials to have arbitrary signs for this correspondence.)

One more question we shall answer now is the following. We have characterised when $\Phi$ is an orthogonal basis, but what Hilbert space is it an orthogonal basis for?

Let us go right ahead with the answer. Define the Paley-Wiener space,

$$
\mathcal{P} \mathcal{W}_{\Omega}(\mathbb{R}):=\left\{\varphi \in \mathrm{L}^{2}(\mathbb{R}): \mathcal{F}[\varphi](\xi)=0 \text { for a.e. } \xi \in \mathbb{R} \backslash \Omega\right\}
$$

where $\Omega$ is the support of $\mathrm{d} \mu$ [20]. Restricting a function's Fourier transform to lie within a subset $\Omega$ of the real line is known as band-limiting, and Paley-Wiener spaces are often referred to as band-limited function spaces and have numerous applications in signal processing.

Before proceeding to prove that this answer is the right one, we have a small disclaimer. From here onwards, we assume that the measure $\mathrm{d} \mu$ in Theorems 6 and 8 is such that polynomials are dense in the space $\mathrm{L}_{2}(\mathbb{R}, \mathrm{d} \mu)$, so that $P$ forms a complete orthonormal basis of the whole space. This is a technical condition, and only in esoteric examples does it fail [3, p. 73]. We will not dwell upon it here, just warn the reader.

Theorem 9 (Orthogonal bases for PW space) Let $\Phi$ be a sequence of orthogonal functions in $\mathrm{L}_{2}(\mathbb{R})$ with a real, skew-symmetric, tridiagonal, irreducible, differentiation matrix. Then $\Phi$ forms an orthogonal basis for the Paley-Wiener space $\mathcal{P} \mathcal{W}_{\Omega}(\mathbb{R})$, where $\Omega$ is the support of $\mathcal{F}\left[\varphi_{0}\right]$.

Proof By Theorem 8, we may write $\varphi_{n}=(-\mathrm{i})^{n} \mathcal{F}^{-1}\left[g \cdot p_{n}\right]$. Recall that $\mathcal{F}\left[\varphi_{0}\right]=g$, so $\Omega$ is the support of $g$. Define the linear map $M$ from the set of polynomials by

$$
M[p]=\mathcal{F}^{-1}[g \cdot p],
$$

for any polynomial $p$. Because $g$ is a mollifier, we have that $g \cdot p \in \mathrm{L}_{2}(\mathbb{R})$, and since the Fourier transform maps $\mathrm{L}_{2}(\mathbb{R})$ itself, we have that $M[p] \in \mathrm{L}_{2}(\mathbb{R})$. Furthermore, for any $\xi \notin \Omega$,

$$
\mathcal{F}[M[p]](\xi)=g(\xi) \cdot p(\xi)=0 \cdot p(\xi)=0,
$$

because $g=\mathcal{F}\left[\varphi_{0}\right]$ and $\Omega$ is its support. Therefore, $M$ maps into $\mathcal{P} \mathcal{W}_{\Omega}(\mathbb{R})$.

Now, consider the inner product after applying $M$ to polynomials $p$ and $q$ :

$$
\int_{-\infty}^{\infty} \overline{M[p](x)} M[q](x) \mathrm{d} x=\int_{\Omega} \overline{g(\xi) p(\xi)} g(\xi) q(\xi) \mathrm{d} \xi=\int_{\Omega} \overline{p(\xi)} q(\xi) \mathrm{d} \mu(\xi) .
$$

We used Parseval's theorem for the first equality. First of all, we see from this that $M$ is a bounded linear operator from polynomials to $\mathcal{P} \mathcal{W}_{\Omega}(\mathbb{R})$. Since we have assumed that polynomials are dense in $\mathrm{L}_{2}(\mathbb{R}, \mathrm{d} \mu)$, we can extend $M$ continuously to the entirety of $\mathrm{L}_{2}(\mathbb{R}, \mathrm{d} \mu)$. Hence, $M$ is in fact an isometry between $\mathrm{L}_{2}(\mathbb{R}, \mathrm{d} \mu)$ and $\mathcal{P} \mathcal{W}_{\Omega}(\mathbb{R})$.

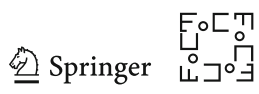


One can readily check that $M$ has a left and right inverse given by $M^{-1}[\varphi](\xi)=$ $\mathcal{F}[\varphi](\xi) / g(\xi)$ for $\xi \in \Omega$ and $M^{-1}[\varphi](\xi)=0$ otherwise. We see that $M$ is an isometric isomorphism between $\mathrm{L}_{2}(\mathbb{R}, \mathrm{d} \mu)$ and $\mathcal{P} \mathcal{W}_{\Omega}(\mathbb{R})$ which maps the orthogonal basis $\left\{(-\mathrm{i})^{n} p_{n}\right\}_{n \in \mathbb{Z}_{+}}$to $\left\{\varphi_{n}\right\}_{n \in \mathbb{Z}_{+}}$. This proves that $\Phi$ is an orthogonal basis for $\mathcal{P} \mathcal{W}_{\Omega}(\mathbb{R})$.

\section{Construction of Orthogonal Systems}

It follows from the analysis of the last section that the right starting point for our analysis is choosing a symmetric Borel measure $\mathrm{d} \mu(\xi)=w(\xi) \mathrm{d} \xi$ in Fourier space, supported either on $\mathbb{R}$ or in an interval of the form $[-a, a], a>0$. This measure determines the mollifier $g$, the constants $\left\{b_{n}\right\}_{n \in \mathbb{Z}_{+}}$and ultimately the orthogonal sequence $\Phi$ in a unique manner. Alternatively, we may choose a positive sequence $\left\{b_{n}\right\}_{n \in \mathbb{Z}_{+}}$and reconstruct the measure $\mathrm{d} \mu$ from (3.4) using Theorem 4 , but this is more problematic because the Favard theorem does not ensure uniqueness of the measure unless, for example, the Carleman criterion is satisfied [3, p. 75]. Yet another alternative is to commence from the seed $\varphi_{0}$ and Fourier transform it, thereby recovering the mollifier and the measure. In the sequel, we restrict ourselves to the first of these three approaches.

We will now demonstrate how to take a symmetric Borel measure $\mathrm{d} \mu(\xi)=w(\xi) \mathrm{d} \xi$ (which is not necessarily normalised to be a probability measure), and produce coefficients $\left\{b_{n}\right\}_{n \in \mathbb{Z}_{+}}$and a seed function $\varphi_{0}$ which generate an orthogonal sequence $\Phi$. Consistent with the last section, we let $g(\xi)=\sqrt{w(\xi)}$ for all $\xi$ in $\Omega$, the support of $\mathrm{d} \mu$ and $g(\xi)=0$ otherwise.

To determine the coefficients $\left\{b_{n}\right\}_{n \in \mathbb{Z}_{+}}$, take any sequence $P=\left\{1, p_{1}, p_{2}, \ldots\right\}$ of orthonormal polynomials for the normalised measure $\mathrm{d} \mu(\xi) / \int \mathrm{d} \mu(\xi)$. The leading coefficients of $p_{1}, p_{2}$ and so on may be negative. Favard's theorem states that $P$ must satisfy a three-term recurrence,

$$
p_{n+1}(\xi)=\left(\alpha_{n}-\beta_{n} \xi\right) p_{n}(\xi)-\gamma_{n} p_{n-1}(\xi), \quad n \in \mathbb{Z}_{+} .
$$

By Lemma 5, $\alpha_{n}=0$ and $\gamma_{n} \beta_{n-1} / \beta_{n}=1$, since $\mathrm{d} \mu$ is a symmetric and positive measure, and $P$ is orthonormal with respect to a probability measure. Now, if we set $b_{n}=\beta_{n}^{-1}$, then

$$
p_{n+1}(\xi)=\frac{1}{b_{n}} \xi p_{n}(\xi)-\frac{b_{n-1}}{b_{n}} p_{n-1}(\xi), \quad n \in \mathbb{Z}_{+}
$$

(where $b_{-1}=0$ ); here, we have used the fact that $\gamma_{n} \beta_{n-1} / \beta_{n}=1$. The signs of the $b_{n}$ 's will be negative if the signs of the leading coefficients of $p_{n}$ and $p_{n+1}$ are the same, and positive otherwise, by Lemma 5.

By the discussion in Sect. 3, the seed for these coefficients $\left\{b_{n}\right\}_{n \in \mathbb{Z}_{+}}$which will lead to an orthogonal system is any scalar multiple of

$$
\varphi_{0}=\frac{\mathcal{F}^{-1}\left[w^{1 / 2}\right]}{\left(\int w(\xi) \mathrm{d} \xi\right)^{\frac{1}{2}}} .
$$


This particular multiple of the seed function will generate an orthonormal sequence by the last part of Theorem 8 .

In some situations, all one has to hand are some orthogonal polynomials $\tilde{P}$ for $\mathrm{d} \mu$ which are not necessarily orthonormal, and have a three-term recurrence of the form

$$
\tilde{p}_{n+1}(\xi)=-\tilde{\beta}_{n} \xi \tilde{p}_{n}(\xi)-\tilde{\gamma}_{n} \tilde{p}_{n-1}(\xi), \quad n \in \mathbb{Z}_{+},
$$

which by Favard's theorem (Theorem 4) satisfies $\tilde{\gamma}_{0}=0$ and $\tilde{\gamma}_{n} \tilde{\beta}_{n-1} / \tilde{\beta}_{n}>0$. From Eq. (3.6) in the proof of Lemma 5, we have

$$
\int \tilde{p}_{n}(\xi)^{2} \mathrm{~d} \mu(\xi)=\tilde{\gamma}_{n} \cdots \tilde{\gamma}_{1} \frac{\tilde{\beta}_{0}}{\tilde{\beta}_{n}} \int \tilde{p}_{0}(\xi)^{2} \mathrm{~d} \mu(\xi)
$$

Therefore, $\tilde{p}_{n}(\xi)=c_{n} p_{n}(\xi)$ where

$$
c_{n}= \pm \sqrt{\tilde{\gamma}_{n} \cdots \tilde{\gamma}_{1} \frac{\tilde{\beta}_{0}}{\tilde{\beta}_{n}} \int \tilde{p}_{0}(\xi)^{2} \mathrm{~d} \mu(\xi)} .
$$

Either sign may be used to obtain all possible orthonormal polynomial sequences $P$. Using this relationship and the three-term recurrence for $\tilde{P}$, we can rewrite the three-term recurrence for $P$ as

$$
p_{n+1}(\xi)=-\frac{c_{n}}{c_{n+1}} \tilde{\beta}_{n} \xi p_{n}(\xi)-\frac{c_{n-1}}{c_{n+1}} \tilde{\gamma}_{n} p_{n-1}(\xi) .
$$

From this, we see that we may take

$$
b_{n}= \pm \sqrt{\frac{\tilde{\gamma}_{n+1}}{\tilde{\beta}_{n+1} \tilde{\beta}_{n}}}, \quad n \in \mathbb{Z}_{+}
$$

and seed function to be any scalar multiple of

$$
\varphi_{0}=\frac{\mathcal{F}^{-1}\left[w^{1 / 2} \cdot p_{0}\right]}{\left(\int p_{0}(\xi)^{2} w(\xi) \mathrm{d} \xi\right)^{\frac{1}{2}}}
$$

As before, this particular multiple generates an orthonormal sequence $\Phi$.

\subsection{Algorithm I}

The simplest approach is to follow (4.7) and (4.8) by consecutive differentiation

$$
\varphi_{n+1}=\frac{1}{b_{n}}\left(\varphi_{n}^{\prime}+b_{n-1} \varphi_{n-1}\right), \quad n \in \mathbb{Z}_{+},
$$

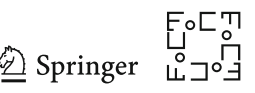


where $b_{-1}=0$. The simplest, alas, is not the best: it produces a sequence of functions, but it then depends on ingenuity and a great deal of careful algebra to identify the underlying construct - if possible-with more familiar functions. A good example is that of Hermite functions (1.5): now $w(x)=\mathrm{e}^{-x^{2}}$; therefore, $g(x)=\mathrm{e}^{-x^{2} / 2}$ and it is possible, using standard formulae for Hermite polynomials, to prove by induction that

$$
\varphi_{n+1}(x)=\sqrt{\frac{2}{n+1}}\left[\varphi_{n}^{\prime}(x)+\sqrt{\frac{n}{2}} \varphi_{n-1}(x)\right]
$$

is consistent with the orthonormal sequence

$$
\varphi_{n}(x)=\frac{1}{\left(2^{n} n ! \pi^{1 / 2}\right)^{1 / 2}} \mathrm{e}^{-x^{2} / 2} \mathrm{H}_{n}(x), \quad n \in \mathbb{Z}_{+},
$$

but this is neither the most natural nor the simplest way of doing so.

\subsection{Algorithm II}

We commence by constructing an orthonormal polynomial system $\left\{p_{n}\right\}_{n \in \mathbb{Z}_{+}}$explicitly. For numerous measures $\mathrm{d} \mu$, such systems are known explicitly; otherwise, we might use the three-term recurrence relation (3.3), perhaps in tandem with the Golub-Welsch algorithm [8]. This is followed by computing (3.7),

$$
\varphi_{n}(x)=(-\mathrm{i})^{n} \mathcal{F}^{-1}\left[w^{1 / 2} \cdot p_{n}\right], \quad n \in \mathbb{Z}_{+} .
$$

Note that by the discussion in Sect. 3, we need to have these polynomials normalised so that their norms in $\mathrm{L}_{2}(\mathbb{R}, \mathrm{d} \mu)$ are all the same, or else we will not obtain a sequence $\Phi$ with a skew-symmetric differentiation matrix. ${ }^{3}$

Returning to Example 1, all we need is to recall that Hermite functions are eigenfunctions of the Fourier transform and that, wishing to obtain an orthonormal sequence,

$$
\int_{-\infty}^{\infty} \mathrm{H}_{n}^{2}(x) \mathrm{e}^{-x^{2}} \mathrm{~d} x=2^{n} n ! \pi^{1 / 2}, \quad n \in \mathbb{Z}_{+}
$$

from Olver et al. [17, Table 18.3.1].

A more interesting example is that of $\mathrm{d} \mu(\xi)=\chi_{[-1,1]}(\xi) \mathrm{d} \xi$ and Legendre polynomials. Now $w(\xi)=\chi_{[-1,1]}(\xi)$ and

$$
(n+1) \mathrm{P}_{n+1}(\xi)=(2 n+1) \xi \mathrm{P}_{n}(\xi)-n \mathrm{P}_{n-1}(\xi)
$$

\footnotetext{
3 The resulting differentiation matrix will clearly be a diagonal similarity transform of a skew-symmetric matrix, which will have the desirable property of having all eigenvalues lie along the imaginary axis, but this is not the aim of our exercise.
} 
from Rainville [18, p. 160], in tandem with (4.7), imply

$$
b_{n}=\frac{n+1}{\sqrt{(2 n+1)(2 n+3)}}, \quad n \in \mathbb{Z}_{+}
$$

we recover Example 2 from Sect. 1. Fourier transforms of Legendre polynomials are known, and we deduce from Olver et al. [17, Eq. 18.17.19] that

$$
\varphi_{n}(x)=\sqrt{\frac{n+\frac{1}{2}}{x}} \mathrm{~J}_{n+\frac{1}{2}}(x), \quad n \in \mathbb{Z}_{+} .
$$

The factor of $\sqrt{n+\frac{1}{2}}$ comes from the normalisation of the Legendre polynomials.

Since $d \mu$ is supported by $[-1,1]$, the above orthonormal sequence is dense in the Paley-Wiener space $\mathcal{P} \mathcal{W}_{[-1,1]}(\mathbb{R})$. Interestingly enough, we did not find a reference to the formula

$$
\int_{-\infty}^{\infty} \mathrm{J}_{m}(x) \mathrm{J}_{n}(x) \frac{\mathrm{d} x}{x}=0, \quad m \neq n, m, n \in \mathbb{Z}_{+},
$$

which follows from our construction, but note that it can be derived directly from Olver et al. [17, Eq. 10.22.6] with little difficulty.

\subsection{Algorithm III}

Formula (2.2) is the starting point to an alternative means to derive the sequence $\Phi$. Recall that

$$
\mathcal{F}\left[\varphi_{0}^{(m-2 \ell)}\right]=(\mathrm{i} \xi)^{m-2 \ell} \mathcal{F}\left[\varphi_{0}\right]
$$

Fourier transforming (2.2) therefore yields

$$
\psi_{n}(\xi)=(-\mathrm{i})^{n} \mathcal{F}\left[\varphi_{0}\right](\xi)=\frac{(-1)^{n} \psi_{0}(\xi)}{b_{0} b_{1} \cdots b_{n-1}} \sum_{\ell=0}^{\lfloor n / 2\rfloor}(-1)^{\ell} \alpha_{n, \ell} \xi^{n-2 \ell}, \quad n \in \mathbb{Z}_{+} .
$$

The polynomial $p_{n}$, being of the same parity as $n$, can be written in the form

$$
p_{n}(\xi)=\sum_{\ell=0}^{\lfloor n / 2\rfloor} p_{n, \ell} \xi^{n-2 \ell}
$$

and, comparing with $\psi_{n}=\psi_{0} p_{n}$, we deduce that

$$
\varphi_{n}(x)=\sum_{\ell=0}^{\lfloor n / 2\rfloor}(-1)^{n-\ell} p_{n, \ell} \varphi_{0}^{(n-2 \ell)}(x), \quad n \in \mathbb{Z}_{+} .
$$

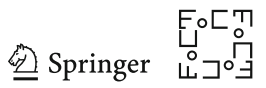


This, together with (2.2), implies that

$$
\alpha_{n, \ell}=(-1)^{n-\ell} b_{1} \cdots b_{n-1} p_{n, \ell}, \quad \ell=0, \ldots,\left\lfloor\frac{n}{2}\right\rfloor
$$

and, more importantly, provides an explicit formula for $\Phi$ in the ubiquitous case when the $p_{n, \ell} \mathrm{s}$ are known.

Inasmuch as (4.12) looks very attractive, we observe that it trades in the computation of Fourier transforms of the $p_{n} \mathrm{~s}$ (as in Algorithm II) for the computation of derivatives of $\varphi_{0}$, which is often fairly complicated. Thus, returning to the example of Legendre polynomials, we deduce from Rainville [18, p. 161] that

$$
p_{n, \ell}=\frac{(-1)^{\ell}\left(\frac{1}{2}\right)_{n-\ell} 2^{n-2 \ell}}{\ell !(n-2 \ell) !}, \quad \ell=0, \ldots\left\lfloor\frac{n}{2}\right\rfloor \text {, }
$$

while

$$
\varphi_{0}(x)=\frac{\mathrm{J}_{\frac{1}{2}}(x)}{\sqrt{x}}=\sqrt{\frac{2}{\pi}} \mathrm{j}_{0}(x),
$$

where $\mathrm{j}_{n}$ is the (entire) spherical Bessel function. We deduce that

$$
\varphi_{n}(x)=(-1)^{n} \sum_{\ell=0}^{\lfloor n / 2\rfloor} \frac{\left(\frac{1}{2}\right)_{n-\ell} 2^{n-2 \ell}}{\ell !(n-2 \ell) !} \mathrm{j}_{0}^{(n-2 \ell)}(x), \quad n \in \mathbb{Z}_{+} .
$$

It is far from straightforward to prove that (4.13) is identical to (4.11), e.g. iterating [17, Eq. 10.51.2].

\section{Systems of Quasi-polynomials}

The normalised Fourier transforms $\psi_{n}$ from Sect. 3 were all of the form $g p_{n}$, with a mollifier $g=\sqrt{w}$ and orthogonal polynomials $p_{n}$, each of degree $n$. Similar pattern is displayed by the functions $\Phi$ in the special case of Hermite functions (Example 1 from Sect. 2). In general, we say that $\left\{f_{n}\right\}_{n \in \mathbb{Z}_{+}}$is a sequence of quasi-polynomials if each $f_{n}$ is a multiple of a function $G$ and a polynomial $q_{n}$ of degree $n$-thus, disregarding scaling, for Hermite functions we have $G(x)=\mathrm{e}^{-x^{2} / 2}$ and $q_{n}=\mathrm{H}_{n}$. Another example of quasi-polynomial $\Phi$ is the quasi-Hermite functions from Example 3.

Quasi-polynomials are of a very attractive form; hence, it is of interest to explore which orthogonal sets $\Phi$ lend themselves to this representation. We somewhat extend the framework from orthogonality in $L_{2}(\mathbb{R})$ to one in $L_{2}(\Omega, d \nu)$ for an arbitrary measure residing in a real interval $\Omega=[a, b]$-in other words,

$$
\int_{a}^{b} \varphi_{m}(x) \varphi_{n}(x) \mathrm{d} \nu(x)=0, \quad m \neq n, m, n \in \mathbb{Z}_{+},
$$

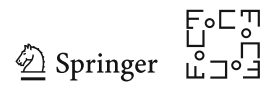


and assume that $\varphi_{n}(x)=G(x) q_{n}(x), \operatorname{deg} q_{n}=n, n \in \mathbb{Z}_{+}$. We further assume that $G^{-1} \in \mathrm{L}_{2}(\Omega)$ and define $\mathrm{d} \eta(x)=G^{-2}(x) \mathrm{d} \nu(x)$ : the orthogonality conditions become

$$
\int_{a}^{b} q_{m}(x) q_{n}(x) \mathrm{d} \eta(x)=0, \quad m \neq n, m, n \in \mathbb{Z}_{+} .
$$

In other words, $\left\{q_{n}\right\}_{n \geq 0}$ is an orthogonal polynomial system corresponding to the measure $\mathrm{d} \eta$. Being orthogonal, the $q_{n} \mathrm{~s}$ obey the three-term recurrence relation

$$
q_{n+1}(x)=\left(\alpha_{n} x-\beta_{n}\right) q_{n}(x)-\gamma_{n} q_{n-1}(x), \quad n \in \mathbb{Z}_{+},
$$

where $\gamma_{0}=0$ and $\gamma_{n}>0, n \in \mathbb{N}$.

Theorem 10 The only system of quasi-polynomials that is orthogonal on a real interval and whose differentiation matrix is real, skew-symmetric, tridiagonal and irreducible is, up to rescaling, the Hermite system (1.5).

Proof Our first observation is that $\varphi_{n}=G q_{n}$, where $q_{n}$ is an $n$th degree polynomial, implies that $\left\{q_{n}\right\}_{n \in \mathbb{Z}_{+}}$is on orthogonal polynomial system with respect to the measure $G^{2}(x) \mathrm{d} x$. Therefore, all zeros of $q_{n}$ are real and distinct.

Since we require a skew-symmetric, tridiagonal, irreducible differentiation matrix, we are within the framework of this paper and the functions $\varphi_{n}=G q_{n}$ obey (2.1). Therefore, assuming $G$ is differentiable,

$$
G^{\prime} q_{n}+G q_{n}^{\prime}=-b_{n-1} G q_{n-1}+b_{n} G q_{n+1}
$$

and, dividing by $G$, we deduce that

$$
\frac{G^{\prime}}{G}=\frac{q_{n}^{\prime}+b_{n-1} q_{n-1}-b_{n} q_{n+1}}{q_{n}}, \quad n \in \mathbb{Z}_{+} .
$$

We have on the right a rational function - a ratio of a polynomial of degree $n+1$ to a polynomial of degree $n$. The poles are distinct, because $q_{n}$ is an orthogonal polynomial, and we deduce that there exist constants $c_{n, 1}, c_{2, n} d_{n, 1}, d_{n, 2}, \ldots, d_{n, n}$ such that

$$
\frac{G^{\prime}}{G}=c_{n, 1}+c_{n, 2} x+\sum_{\ell=1}^{n} \frac{d_{n, \ell}}{x-\zeta_{n, \ell}}
$$

where $\zeta_{n, 1}, \ldots, \zeta_{n, n} \in(a, b)$ are the zeros of $q_{n}$. Note, however, that the left-hand side of (5.2) is independent of $n$-this implies that $c_{n, 1}=c_{1}, c_{n, 2}=c_{2}$ and $d_{n, 1}=$ $\cdots=d_{n, n}=0$-in other words,

$$
G^{\prime}=\left(c_{1}+c_{2} x\right) G
$$

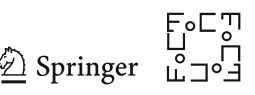


The solution of this ODE is $G(x)=\mathrm{e}^{c_{1} x+c_{2} x^{2}} G(0)$ for some $G(0) \neq 0$; therefore,

$$
\left(c_{1}+c_{2} x\right) q_{n}=q_{n}^{\prime}+b_{n-1} q_{n-1}-b_{n} q_{n+1} .
$$

This is the moment to use the recurrence relation (5.1) to replace $q_{n+1}$ : we obtain

$$
\left(c_{1}+c_{2} x\right) q_{n}=b_{n}\left(\alpha_{n} x+\beta_{n}\right) q_{n}-q_{n}^{\prime}-\left(b_{n-1}+b_{n} \gamma_{n}\right) q_{n-1} .
$$

We have polynomials of degree $n+1$ on both sides, and comparing the coefficient of $x^{n+1}$, we deduce that $c_{2}=b_{n} \alpha_{n}$ - the equality reduces to

$$
c_{1} q_{n}=b_{n} \beta_{n} q_{n}-q_{n}^{\prime}-\left(b_{n-1}+b_{n} \gamma_{n}\right) q_{n-1}
$$

We now have on the left and the right polynomials of degree $n$ and compare the coefficients of $x^{n}$ there-this yields $c_{1}=b_{n} \beta_{n}$ and we are left with

$$
q_{n}^{\prime}=-\left(b_{n-1}+b_{n} \gamma_{n}\right) q_{n-1}, \quad n \in \mathbb{Z}_{+} .
$$

We next recall the classical theorem of Hahn [9], namely that the only orthogonal polynomial systems whose derivatives are also orthogonal are Jacobi, Laguerre and Hermite polynomials. ${ }^{4}$ Specifically,

$$
\begin{aligned}
\frac{\mathrm{dP}_{n}^{(\alpha, \beta)}(x)}{\mathrm{d} x} & =\frac{\alpha+\beta+n+1}{2} \mathrm{P}_{n-1}^{(\alpha+1, \beta+1)}(x), \\
\frac{\mathrm{dL}^{(\alpha)}(x)}{\mathrm{d} x} & =-\mathrm{L}_{n-1}^{(\alpha+1)}(x), \\
\frac{\mathrm{dH}_{n}(x)}{\mathrm{d} x} & =2 n \mathrm{H}_{n-1}(x) .
\end{aligned}
$$

We need, however, more: to be consistent with (5.3), we want $q_{n}^{\prime}$ to be a constant multiple of $q_{n-1}$, therefore orthogonal with respect to the same measure-and this is the case only once (up to a multiplicative constant) $q_{n}=\mathrm{H}_{n}$. Then, the Hermite measure being determinate [3, p. 58], necessarily for orthogonality $G(x)=\mathrm{e}^{-x^{2} / 2}$ and the proof is complete.

\section{Examples}

In this section, we present a number of examples, grouped into two: orthogonality in the Paley-Wiener space $\mathcal{P} \mathcal{W}_{[-1,1]}(\mathbb{R})$ and orthogonality in $\mathrm{L}_{2}(\mathbb{R})$. Not all these examples are completely worked out, they are often no more than pointers towards interesting instances of orthogonal systems $\Phi$, calling for further research.

\footnotetext{
4 Some versions of this theorem mention also Bessel polynomials [3, p. 151], but they are not orthogonal with respect to a positive-definite measure, and anyway, their inclusion does not change our argument.
} 
A word about terminology: once a system of orthogonal functions $\Phi$ originates in a named set of orthogonal polynomials, we call them transformed named functions. For instance, if $\Phi$ originated in the fictitious Bloggs Polynomials, we call them transformed Bloggs functions.

\subsection{Paley-Wiener Spaces}

\subsubsection{Ultraspherical Polynomials}

We set $\mathrm{d} \mu(x)=\chi_{[-1,1]}(x)\left(1-x^{2}\right)^{\alpha} \mathrm{d} x$, where $\alpha>-1$ : the underlying ultraspherical polynomials $\mathrm{P}_{n}^{(\alpha, \alpha)}$ obey the three-term recurrence relation

$\mathrm{P}_{n+1}^{(\alpha, \alpha)}(x)=\frac{(2 n+2 \alpha+1)(n+\alpha+1)}{(n+1)(n+2 \alpha+1)} x \mathrm{P}_{n}^{(\alpha, \alpha)}(x)-\frac{(n+\alpha)(n+\alpha+1)}{(n+1)(n+2 \alpha+1)} \mathrm{P}_{n-1}^{(\alpha, \alpha)}(x)$

from Rainville [18, p. 263]. Special case are Legendre polynomials $\mathrm{P}_{n}(\alpha=0)$, which we have already considered in Sect. 4, and (under different scaling) Chebyshev polynomials of the first kind, $\mathrm{T}_{n}$, and second kind, $\mathrm{U}_{n}$, for $\alpha=-\frac{1}{2}$ and $\alpha=\frac{1}{2}$, respectively. Up to another rescaling, ultraspherical polynomials for $\alpha>-\frac{1}{2}$ are the same as Gegenbauer polynomials [18, p. 276].

The mollifier is $g(x)=\chi_{[-1,1]}(x)\left(1-x^{2}\right)^{\alpha / 2}$, and according to (4.7),

$$
b_{n}=\frac{1}{2} \sqrt{\frac{(n+1)(n+2 \alpha+1)}{\left(n+\alpha+\frac{1}{2}\right)\left(n+\alpha+\frac{3}{2}\right)}}, \quad n \in \mathbb{Z}_{+} .
$$

Note that for $\alpha=0$ this is consistent with (4.10).

Very lengthy algebra of little intrinsic interest yields

$$
\varphi_{0}(x)=\frac{1}{\sqrt{2 \pi}} \int_{-1}^{1}\left(1-\xi^{2}\right)^{\alpha / 2} \mathrm{e}^{\mathrm{i} x \xi} \mathrm{d} \xi=\frac{2^{\alpha / 2} \Gamma\left(1+\frac{\alpha}{2}\right)}{x^{\frac{1}{2}+\frac{\alpha}{2}}} \mathrm{~J}_{\frac{1}{2}+\frac{\alpha}{2}}(x),
$$

and this can be extended to other $\varphi_{n} \mathrm{~s}$ with Algorithm II. $^{5}$ Intriguingly, while $\varphi_{1}$ is a scalar multiple of $\mathrm{J}_{\frac{3}{2}+\frac{\alpha}{2}}(x) / x^{\frac{1}{2}+\frac{\alpha}{2}}$, consistently with the special case of Legendre polynomials, this neat representation is no longer true for $\varphi_{2}$ and beyond.

In Fig. 1, we display the functions $\varphi_{n}, n=0, \ldots, 5$, corresponding to the Chebyshev measure of the second kind, $\mathrm{d} \mu(\xi)=\chi_{[-1,1]}(\xi)\left(1-\xi^{2}\right)^{1 / 2} \mathrm{~d} \xi$-in other words, $\alpha=\frac{1}{2}$ and $b_{n} \equiv \frac{1}{2}$. The functions $\varphi_{n}$ are linear combinations (with polynomial coefficients) of Bessel functions.

\footnotetext{
5 Section 18.17 of Olver et al. [17] lists explicitly the Fourier transforms of all classical orthogonal polynomials, inclusive of $\mathrm{P}_{n}^{(\alpha, \alpha)}$. Unfortunately, this is irrelevant for us (except when $\alpha=0$ ) because of the need to square root the weight function.
}

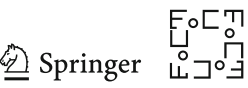




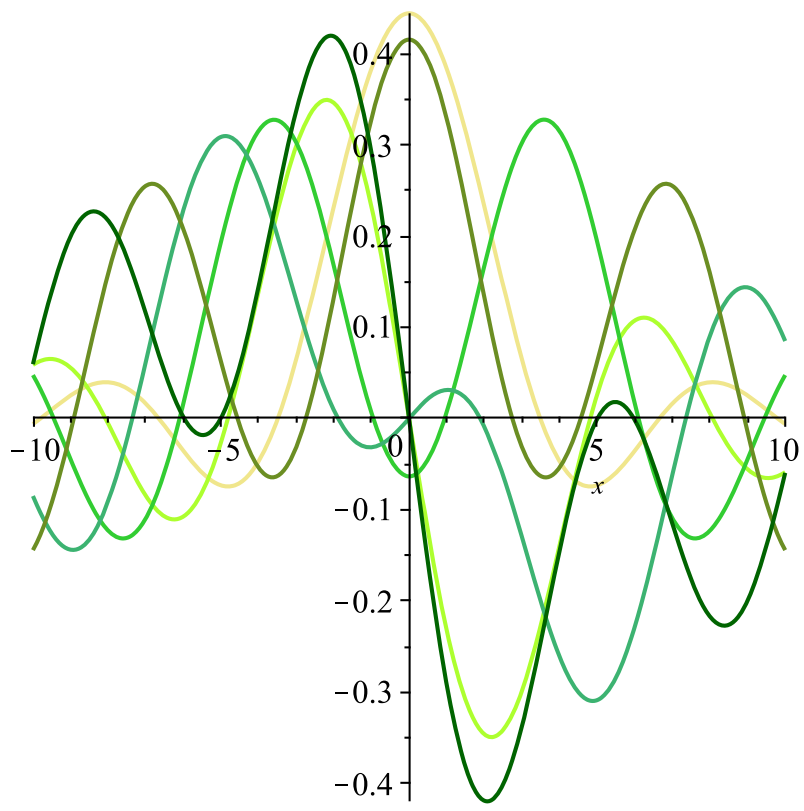

Fig. 1 Transformed Chebyshev functions of the second kind $\varphi_{n}$ for $n=0, \ldots, 5$ : darker colour denotes greater $n$ (Color figure online)

\subsubsection{Konoplev Polynomials}

Let $\alpha, \beta>-1$ and consider $\mathrm{d} \mu(\xi)=\chi_{[-1,1]}(\xi)|\xi|^{2 \beta+1}\left(1-\xi^{2}\right)^{\alpha} \mathrm{d} \xi$. Corresponding orthogonal polynomial systems $\left\{S_{n}^{(\alpha, \beta)}\right\}_{n \geq 0}$ have been originally considered by Szegó [21] in the case $\alpha=0$, and the general case is due to Konoplev [14]. They can be conveniently expressed in terms of Jacobi polynomials,

$$
\mathrm{S}_{2 n}^{(\alpha, \beta)}(\xi)=\mathrm{P}_{n}^{(\alpha, \beta)}\left(2 \xi^{2}-1\right), \quad \mathrm{S}_{2 n+1}^{(\alpha, \beta)}(\xi)=\xi \mathrm{P}_{n}^{(\alpha, \beta+1)}\left(2 \xi^{2}-1\right), \quad n \in \mathbb{Z}_{+},
$$

while the recurrence relation for monic Konoplev polynomials $\left\{\check{\mathbf{S}}_{n}^{(\alpha, \beta)}\right\}_{n \geq 0}$ is

$$
\check{\mathrm{S}}_{n+1}^{(\alpha, \beta)}(\xi)=\xi \check{\mathrm{S}}_{n}^{(\alpha, \beta)}(\xi)-\gamma_{n} \check{\mathrm{S}}_{n-1}^{(\alpha, \beta)}(\xi),
$$

where

$$
\gamma_{2 n}=\frac{n(n+\alpha)}{(2 n+\alpha+\beta)(2 n+\alpha+\beta+1)}, \quad \gamma_{2 n+1}=\frac{(n+\beta+1)(n+\alpha+\beta+1)}{(2 n+\alpha+\beta+1)(2 n+\alpha+\beta+2)}
$$

from Chihara [3, p. 221]. Of course, $b_{n}=\sqrt{\gamma_{n+1}}$ and note that this reduces to the ultraspherical case from Sect. 6.1.1 once $\beta=-\frac{1}{2}$. 
The calculation of $\varphi_{0}$ is long and complicated: the outcome is

$$
\begin{aligned}
\varphi_{0}(x)= & 2 \Gamma(\alpha+1)\left\{\Gamma\left(\alpha+\beta+\frac{5}{2}\right) \Gamma(\beta+1) \sin (\pi \beta)_{1} F_{2}\left[\begin{array}{l}
\beta+1 ; \\
\frac{1}{2}, \alpha+\beta+2 ;
\end{array}-\frac{x^{2}}{4}\right]\right. \\
& \left.+\Gamma(2+\alpha+\beta) \Gamma\left(\beta+\frac{3}{2}\right) \cos (\pi \beta) x_{1} F_{2}\left[\begin{array}{l}
\beta+\frac{3}{2} ; \\
\frac{3}{2}, \alpha+\beta+\frac{5}{2} ;-\frac{x^{2}}{4}
\end{array}\right]\right\} .
\end{aligned}
$$

In the most interesting special case $\alpha=0$, this can be simplified. Given $a, b>-1$,

$$
\begin{aligned}
{ }_{1} F_{2}\left[\begin{array}{l}
a ; \\
\left.a+1, b+1 ;-\frac{x^{2}}{4}\right]
\end{array}\right. & =\sum_{\ell=0}^{\infty}(-1)^{\ell} \frac{(a)_{\ell}}{\ell !(a+1)_{\ell}(b+1)_{\ell}} \frac{x^{2 \ell}}{4^{\ell}} \\
& =a \sum_{\ell=0}^{\infty} \frac{(-1)^{\ell}}{\ell !(b+1)_{\ell} 4^{\ell}} \frac{x^{2 \ell}}{a+\ell} \\
& =a x^{-2 a} \int_{0}^{x} \tau^{2 a-1} \sum_{\ell=0}^{\infty} \frac{1}{\ell !(b+1)_{\ell}}\left(-\frac{\tau^{2}}{4}\right)^{\ell} \mathrm{d} \tau \\
& =a 2^{b} \Gamma(b+1) x^{-2 a} \int_{0}^{x} \tau^{2 a-b-1} \mathrm{~J}_{b}(\tau) \mathrm{d} \tau .
\end{aligned}
$$

Substitution in (6.1) and lengthy algebra result in a far simpler formula,

$$
\varphi_{0}(x)=2 \Gamma(\beta+2) \Gamma\left(\beta+\frac{5}{2}\right) x^{-2 \beta-2} \int_{0}^{x} \tau^{2 \beta+1} \sin (\tau+\pi \beta) d \tau .
$$

Subsequent $\varphi_{n} \mathrm{~s}$ (obtained by the brute-force Algorithm I) do not appear to possess any recognisable structure.

\subsection{Systems Dense in $L_{2}(\mathbb{R})$}

While orthogonal systems $\Phi$ which are dense in a Paley-Wiener space have, as far as we can see, limited applications, this is hardly the case with systems dense in $L_{2}(\mathbb{R})$, which have an immediate relevance to spectral methods, along the lines of Sect. 1.

\subsubsection{Generalised Hermite Polynomials}

Hermite functions are a familiar tool and the most important example of an orthogonal system that shares all the welcome properties sought in this paper: a skew-symmetric, tridiagonal, irreducible differentiation matrix and density in $\mathrm{L}_{2}(\mathbb{R})$. It is interesting, though, to generalise them in line with the Szegó [22, Problem 25] generalisation of Hermite polynomials.

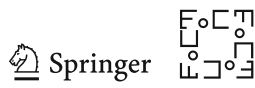


Letting $\eta>-\frac{1}{2}$, we denote by $\mathrm{H}_{n}^{(\eta)}$ the polynomials orthogonal with respect to the measure

$$
\mathrm{d} \mu(\xi)=|\xi|^{2 \eta} \mathrm{e}^{-\xi^{2}} \mathrm{~d} \xi, \quad \xi \in \mathbb{R}
$$

They obey the three-term recurrence relation

$$
\mathrm{H}_{n+1}^{(\eta)}(\xi)=2 \xi \mathrm{H}_{n}^{(\eta)}(\xi)-2\left(n+\theta_{n}\right) \mathrm{H}_{n-1}^{(\eta)}(\xi)
$$

where

$$
\theta_{n}= \begin{cases}0, & n \text { even } \\ 2 \eta, & n \text { odd }\end{cases}
$$

from Chihara [3, pp. 156-157]. They can be represented explicitly in terms of Laguerre polynomials,

$$
\mathrm{H}_{2 n}^{(\eta)}(\xi)=(-1)^{n} 2^{2 n} n ! \mathrm{L}_{n}^{\left(\eta-\frac{1}{2}\right)}\left(\xi^{2}\right), \quad \mathrm{H}_{2 n+1}^{(\eta)}(\xi)=(-1)^{n} 2^{2 n+1} n ! \mathrm{L}_{n}^{\left(\eta+\frac{1}{2}\right)}\left(\xi^{2}\right) .
$$

The coefficients are thus

$$
b_{2 n}=\sqrt{n+\eta+1}, \quad b_{2 n+1}=\sqrt{n+1}, \quad n \in \mathbb{Z}_{+},
$$

while the mollifier is $g(\xi)=|\xi|^{\eta} \mathrm{e}^{-\xi^{2} / 2}$. Assuming that $\eta$ is not an odd integer, it follows from (4.8) that $\varphi_{0}(x)=\frac{1}{\sqrt{2 \pi}} \int_{-\infty}^{\infty}|\xi|^{\eta} \mathrm{e}^{-\xi^{2} / 2+\mathrm{i} x \xi} \mathrm{d} \xi=\frac{\mathrm{e}^{-x^{2} / 4}}{2 \cos \frac{\pi \eta}{2}}\left[\mathrm{U}\left(-\eta-\frac{1}{2} x\right)+\mathrm{U}\left(-\eta-\frac{1}{2},-x\right)\right]$

where U are Weber parabolic cylinder functions,

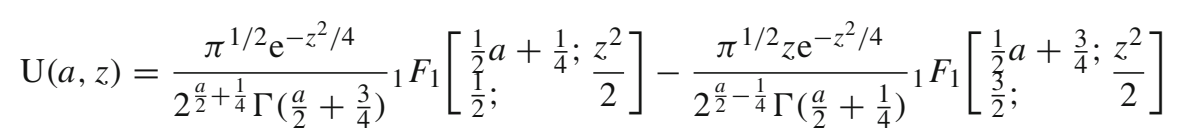

from Olver et al. [17, Eq. 12.7.12]. It follows that

$$
\mathrm{U}\left(-\eta-\frac{1}{2} x\right)+\mathrm{U}\left(-\eta-\frac{1}{2},-x\right)=\frac{\pi^{1 / 2} 2^{\eta / 2+1} \mathrm{e}^{-z^{2} / 4}}{\Gamma\left(\frac{1}{2}-\frac{\eta}{2}\right)}{ }_{1} F_{1}\left[\begin{array}{l}
-\frac{\eta}{2} ; \\
\frac{1}{2} ;
\end{array}\right]
$$

and we simplify,

$$
\varphi_{0}(x)=c_{0} \mathrm{e}^{-x^{2} / 2}{ }_{1} F_{1}\left[\begin{array}{l}
-\frac{1}{2} \eta ; \\
\frac{1}{2} ;
\end{array} \frac{x^{2}}{2}\right], \quad c_{0}=\frac{1}{\cos \frac{\pi \eta}{2}} \frac{\pi^{1 / 2} 2^{\eta / 2}}{\Gamma\left(\frac{1}{2}-\frac{\eta}{2}\right)} .
$$


According to Olver et al. [17, Eq. 13.3.16],

$$
\frac{\mathrm{d}^{m}}{\mathrm{~d} x^{m}}{ }_{1} F_{1}\left[\begin{array}{l}
a ; \\
b ;
\end{array}\right]=\frac{(a)_{m}}{(b)_{m}}{ }_{1} F_{1}\left[\begin{array}{l}
a+m ; \\
b+m ;
\end{array}\right], \quad m \in \mathbb{Z}_{+} .
$$

Using this, it is easy (though laborious) to prove by induction that

$$
\begin{gathered}
\varphi_{0}^{(2 m)}(x)=c_{0}(-1)^{m} 2^{m}\left(\frac{1}{2}+\frac{\eta}{2}\right)_{m} \mathrm{e}^{-x^{2} / 2}{ }_{1} F_{1}\left[\begin{array}{l}
-\frac{1}{2} \eta-m ; \\
\frac{1}{2} ;
\end{array} ;\right. \\
\varphi_{0}^{(2 m+1)}(x)=c_{0}(-1)^{m} 2^{m}\left(\frac{3}{2}+\frac{\eta}{2}\right)_{m} x \mathrm{e}^{-x^{2} / 2}{ }_{1} F_{1}\left[\begin{array}{l}
-\frac{\eta}{2}-m ; \\
\frac{3}{2} ;
\end{array}\right] .
\end{gathered}
$$

Since [e.g. using (6.2)]

$$
\begin{gathered}
\mathrm{H}_{2 n}^{(\eta)}(x)=\sum_{\ell=0}^{n}\left(\begin{array}{l}
n \\
\ell
\end{array}\right)\left(-n-\eta+\frac{1}{2}\right)_{\ell} x^{2 n-2 \ell}, \\
\mathrm{H}_{2 n+1}^{(\eta)}(x)=\sum_{\ell=0}^{n}\left(\begin{array}{l}
n \\
\ell
\end{array}\right)\left(-n-\eta-\frac{1}{2}\right)_{\ell} x^{2 n+1-2 \ell},
\end{gathered}
$$

we now have all that is required to implement Algorithm III: the outcome is

$$
\begin{aligned}
\varphi_{2 n}(x) & =c_{0} \frac{(-1)^{n}\left(\frac{1}{2}+\frac{\eta}{2}\right)_{n}}{2^{n}} \mathrm{e}^{-x^{2} / 2} \sum_{\ell=0}^{n}\left(\begin{array}{l}
n \\
\ell
\end{array}\right) \frac{(-1)^{\ell}\left(-n-\eta+\frac{1}{2}\right)_{\ell}}{2^{\ell}\left(-n+\frac{1}{2}-\frac{\eta}{2}\right)_{\ell}} F_{1}\left[\begin{array}{l}
-n+\ell-\frac{\eta}{2} ; \\
\frac{1}{2} ;
\end{array}\right], \\
\varphi_{2 n+1}(x) & =c_{0} \frac{(-1)^{n}\left(\frac{3}{2}+\frac{\eta}{2}\right)_{n}}{2^{n}} x \mathrm{e}^{-x^{2} / 2} \sum_{\ell=0}^{n}\left(\begin{array}{l}
n \\
\ell
\end{array}\right) \frac{(-1)^{\ell}\left(-n-\eta-\frac{1}{2}\right)_{\ell}}{2^{\ell}\left(-n-\frac{1}{2} \eta-\frac{1}{2}\right)_{\ell}}{ }_{1} F_{1}\left[\begin{array}{l}
-n+\ell-\frac{\eta}{2} ; \\
\frac{3}{2} ;
\end{array}\right] .
\end{aligned}
$$

Once $\eta$ is an even integer, the above expansions terminate; however, only for $\eta=0$ we recover quasi-polynomials, in line with Theorem 11 . For example, for $\eta=2$

$$
\varphi_{2 m}(x)=c_{2 m} \mathrm{e}^{-x^{2} / 2} \sum_{k=0}^{m+1}(2 m+2-k) \frac{(-1)^{k}}{k !\left(\frac{1}{2}\right)_{k}} x^{2 k}
$$

it is a multiple of $G(x)=\mathrm{e}^{-x^{2} / 2}$ by a polynomial of degree $2 m+2$ and hence does not fit the definition of quasi-polynomials.

\subsubsection{Carlitz Polynomials}

The original Carlitz polynomials [2] have been defined with respect to a measure supported by a vertical line in the complex plane: letting $\lambda \in(-1,1)$, we consider polynomials

$$
\Omega_{n}^{(\lambda)}(z)=\frac{(-1)^{n}(1+\lambda)_{n} n !}{2^{m}\left(\frac{1}{2}\right)_{m}}{ }_{3} F_{2}\left[\begin{array}{l}
-n, n+1,1+z ; 1 \\
1,1+\lambda ;
\end{array}\right], \quad n \in \mathbb{Z}_{+},
$$

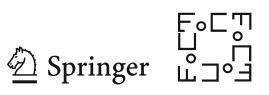


which obey the orthogonality conditions

$$
\int_{-c-\mathrm{i} \infty}^{-c+\mathrm{i} \infty} \frac{\Omega_{m}^{(\lambda)}(z) \Omega_{n}^{(\lambda)}(z) \mathrm{d} z}{\sin (\pi z) \sin (\pi(z-\lambda))}=\frac{2 \mathrm{i} \lambda}{\sin \pi \lambda} \frac{(-1)^{m} m !^{2}(1+\lambda)_{m}(1-\lambda)_{m}}{(2 m+1)(m-1) !^{2}} \delta_{m, n},
$$

where $c \in(0,1)$ and $m ! !=\prod_{j=0}^{m-1}(2 j+1)=(2 m+1) ! /\left(2^{m} m !\right)$ is the double factorial. Original interest in such polynomials sprung from the connection between their moments and the classical Bernoulli polynomials [3, pp. 192-193].

We can recover orthogonality on the real line by a change of variables,

$$
\mathrm{G}_{n}^{(\lambda)}(\xi)=(-\mathrm{i})^{n} \Omega_{n}^{(\lambda)}\left(\frac{\lambda-1+\mathrm{i} \xi}{2}\right), \quad n \in \mathbb{Z}_{+},
$$

whereby

$$
\mathrm{d} \mu(\xi)=\frac{\mathrm{d} \xi}{\cos \pi \lambda+\cosh \pi \xi}, \quad \xi \in \mathbb{R}
$$

The three-term recurrence relation for monic Carlitz polynomials being

$$
\mathrm{G}_{n+1}^{(\lambda)}(\xi)=\xi \mathrm{G}_{n}^{(\lambda)}(\xi)-\frac{n^{2}\left(n^{2}-\lambda^{2}\right)}{4 n^{2}-1} \mathrm{G}_{n-1}^{(\lambda)}(x),
$$

we have

$$
b_{n}=(n+1) \sqrt{\frac{(n+1)^{2}-\lambda^{2}}{(2 n+1)(2 n+3)}}, \quad n \in \mathbb{Z}_{+} .
$$

Unfortunately, the closed form of the seed

$$
\varphi_{0}(x)=\frac{1}{\sqrt{2 \pi}} \int_{-\infty}^{\infty} \frac{\mathrm{e}^{\mathrm{i} x \xi} \mathrm{d} \xi}{\cos \pi \lambda+\cosh \pi \xi}, \quad x \in \mathbb{R}
$$

is in general unknown for $\lambda \in(-1,1)$. For $\lambda=0$, though, it can be computed. The measure reduces to

$$
\mathrm{d} \mu(\xi)=\frac{2 \mathrm{~d} \xi}{\cosh ^{2}(\pi \xi)}, \quad \xi \in \mathbb{R}
$$

and its moments $\mu_{n}=\int_{-\infty}^{\infty} \xi^{n} \mathrm{~d} \mu(\xi)$ are

$$
\mu_{2 m}=(-1)^{m}\left(\frac{2}{\pi}\right)^{3 / 2} \mathrm{~B}_{2 m}\left(\frac{1}{2}\right), \quad \mu_{2 m+1}=0, \quad m \in \mathbb{Z}_{+},
$$

where the $\mathrm{B}_{n} \mathrm{~s}$ are Bernoulli polynomials. 
We next compute the seed: since $\sqrt{1+\cosh \pi \xi}=\sqrt{2} \cosh \frac{\pi \xi}{2}$, we have

$$
\varphi_{0}(x)=\frac{1}{2 \sqrt{\pi}} \int_{-\infty}^{\infty} \frac{\mathrm{e}^{\mathrm{i} x \xi} \mathrm{d} \xi}{\cosh \frac{\pi \xi}{2}}
$$

According to Olver et al. [17, Table 1.14(vii)],

$$
\mathcal{F}^{-1}\left(\frac{\cosh a \xi}{\cosh \xi}\right)=\sqrt{\frac{2}{\pi}} \frac{\cos \frac{a}{2} \cosh \frac{x}{2}}{\cos a+\cosh x}, \quad|a|<\pi .
$$

It follows at once by trivial rescaling that

$$
\varphi_{0}(x)=2 \sqrt{\frac{2}{\pi}} \frac{\cosh x}{1+\cosh 2 x}=\sqrt{\frac{2}{\pi}} \frac{1}{\cosh x} .
$$

Dropping the factor $(2 / \pi)^{1 / 2}$, we thus have

$$
\begin{aligned}
& \varphi_{0}(x)=\frac{1}{\cosh x} \\
& \varphi_{1}(x)=-\sqrt{3} \frac{\sinh x}{\cosh 2 x} \\
& \varphi_{2}(x)=\frac{\sqrt{5}}{2}\left(\frac{2}{\cosh x}-\frac{3}{\cosh ^{3} x}\right), \\
& \varphi_{3}(x)=\frac{\sqrt{7}}{2} \sinh x\left(\frac{2}{\cosh ^{2} x}-\frac{5}{\cosh ^{4} x}\right), \\
& \varphi_{4}(x)=\frac{\sqrt{9}}{8}\left(\frac{8}{\cosh x}-\frac{40}{\cosh ^{3} x}+\frac{35}{\cosh ^{5} x}\right)
\end{aligned}
$$

and so on. We can certainly discern some structure, although this issue is not pursued further in the current paper. Further structure is revealed in Fig. 2: each $\varphi_{n}$ appears to have $n$ real zeros which interlace.

Although we do not pursue further this theme, transformed Carlitz functions might well be a good subject for further investigation. As things stand, we just record them as an example of an orthogonal system $\Phi$ of an entirely different flavour than, say, Hermite and transformed generalised Hermite functions.

\subsubsection{Freud Polynomials}

Given $\eta>-\frac{1}{2}$ and an even function $Q \in \mathrm{C}^{\infty}(\mathbb{R})$, such that $Q(x) \geq 0$ for $|x| \gg 1$, orthogonal polynomials with respect to $|x|^{\eta} \mathrm{e}^{-Q(x)} \mathrm{d} x$ are called Freud polynomials $[15,23]$. Hermite polynomials form the simplest example, and other familiar cases are $Q(x)=x^{4}-t x^{2}$ for some $t \in \mathbb{R}$ and $Q(x)=|x|$ (whereby smoothness fails at the origin without any ill effects).

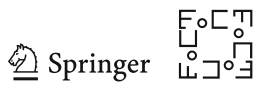




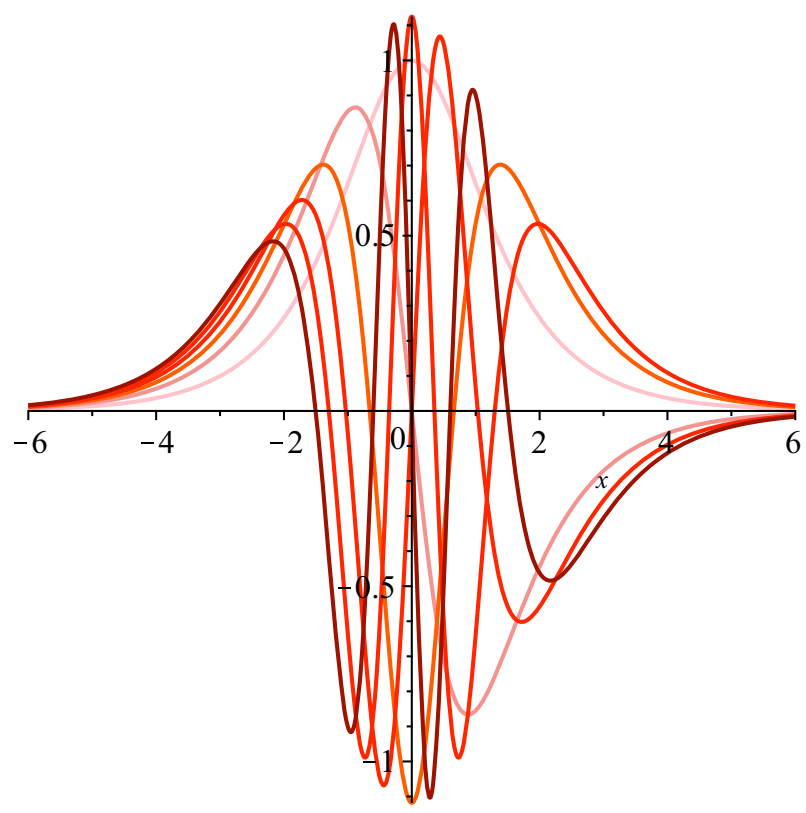

Fig. 2 Transformed Carlitz functions $\varphi_{n}$ for $n=0, \ldots, 5$ : darker colour denotes greater $n$ (Color figure online)

General rules governing the asymptotic behaviour of recurrence coefficients for Freud polynomials have been a major open problem in the theory of orthogonal polynomials which has been solved in a celebrated paper by Fokas et al. [7]. (See also Deift et al. [5].) This has led to a burgeoning body of work, combining theory of orthogonal polynomials, the Riemann-Hilbert transform, Painlevé equations and random matrix theory. Yet, even in the simplest non-trivial case, $\mathrm{d} \mu(x)=\mathrm{e}^{-x^{4}} \mathrm{~d} x$, explicit expressions of recurrence coefficients, the one item of information necessary for the formation of $\Phi$, are unknown.

Yet, the sequence $\left\{\gamma_{m}\right\}_{m \in \mathbb{Z}_{+}}$can in this case be generated recursively from

$$
\gamma_{0}=0, \quad \gamma_{1}=\frac{\Gamma\left(\frac{3}{4}\right)}{\Gamma\left(\frac{1}{4}\right)}, \quad 4 \gamma_{n}\left(\gamma_{n-1}+\gamma_{n}+\gamma_{n+1}\right)=n, \quad n \in \mathbb{N}
$$

from Shohat [19]. The string relation (6.3) had been extended by Magnus [16] to the measure $\mathrm{d} \mu(x)=\mathrm{e}^{-x^{4}+t x^{2}} \mathrm{~d} x$,

$$
\gamma_{n}\left(\gamma_{n-1}+\gamma_{n}+\gamma_{n+1}\right)+2 t \gamma_{n}=n, \quad n \in \mathbb{N},
$$

where

$$
\gamma_{0}=0, \quad \gamma_{1}=\frac{|t|\left[\mathrm{K}_{\frac{3}{4}}\left(\frac{t^{2}}{8}\right)-\mathrm{K}_{\frac{1}{4}}\left(\frac{t^{2}}{8}\right)\right]}{4 \mathrm{~K}_{\frac{1}{4}}\left(\frac{t^{2}}{8}\right)}
$$




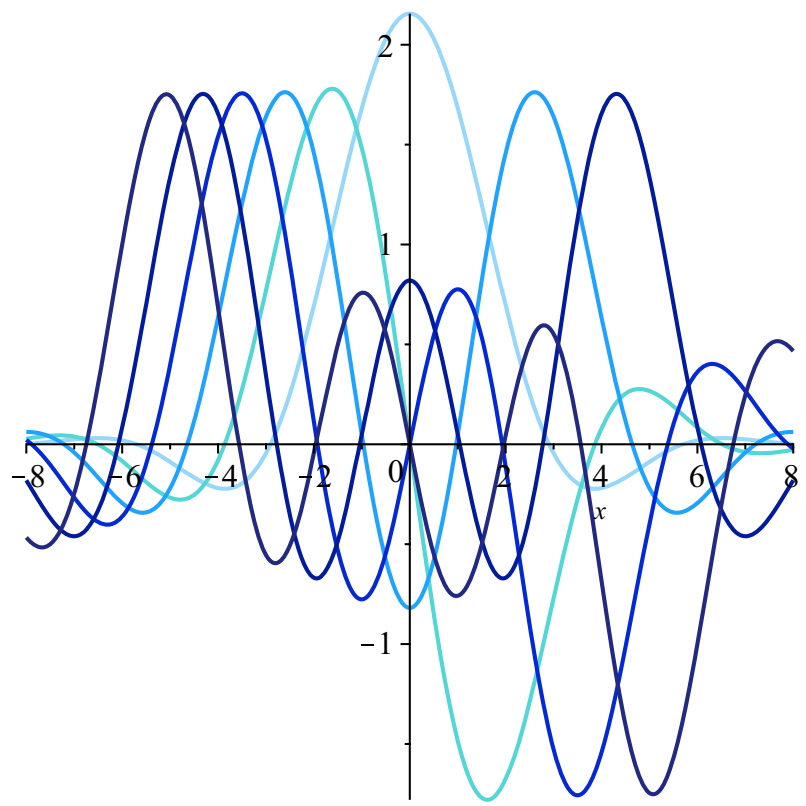

Fig. 3 Transformed Freud functions $\varphi_{n}$ (with $t=\eta=0$ ) for $n=0, \ldots, 5$ : darker colour denotes greater $n$ (Color figure online)

and $\mathrm{K}_{v}$ is a modified Bessel function of the second kind (also known as Macdonald function). Further generalisation to $\mathrm{d} \mu(x)=|x|^{\eta} \mathrm{e}^{-x^{4}+t x^{2}} \mathrm{~d} x$ has been presented in Clarkson and Jordaan [4], who have also given explicit relations of recurrence coefficients in terms of Wronskians related to the Painlevé IV equation. ${ }^{6}$

At least in principle, we may use either (6.3) or (6.4) to calculate as many coefficients $\gamma_{n}$ (therefore also $b_{n}=\sqrt{\gamma_{n+1}}$ ) as required. The other ingredient in constructing $\Phi$ is $\varphi_{0}$, the (scaled) inverse Fourier transform of the square root of the weight function. For $t=0$ [corresponding to (6.3)], we have

$$
\varphi_{0}(x)=\frac{2^{\frac{3}{4}}}{4 \Gamma\left(\frac{3}{4}\right)}\left\{2 \pi_{0} F_{2}\left[\frac{-}{\frac{1}{2}} ; \frac{3}{4} ; \frac{x^{4}}{128}\right]-x^{2} \Gamma^{2}\left(\frac{3}{4}\right)_{0} F_{2}\left[\frac{5}{\frac{5}{4}}, \frac{3}{2} ; \frac{x^{4}}{128}\right]\right\},
$$

while for $t \neq 0$ the explicit form of the seed $\varphi_{0}$ is unknown.

In Fig. 3, we have plotted the first six functions $\varphi_{m}$ for the above transformed Freud functions. Inasmuch as they look quite complicated-linear combinations with polynomial coefficients of ${ }_{0} F_{2}$ hypergeometric functions-they display fairly regular behaviour. It is too early to guess what the features are (in particular insofar as approximation theory is concerned) of this orthogonal system and whether it is of any importance in the context of spectral methods on the real line.

\footnotetext{
6 These explicit coefficients, unfortunately, cannot be computed easily and rapidly.

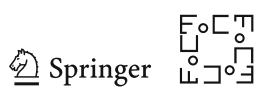




\section{Conclusions and Pointers to Future Research}

In this paper, we have completely characterised orthogonal systems $\Phi$ in $\mathrm{L}_{2}(\mathbb{R})$ whose differentiation matrix is real, skew-symmetric, tridiagonal and irreducible. In essence, to every symmetric probability measure $\mathrm{d} \mu(\xi)=w(\xi) \mathrm{d} \mu(\xi)$ there corresponds an essentially unique $\Phi$, which is dense in $L_{2}(\mathbb{R})$ if the support of $d \mu$ is the real line or dense in an appropriate Paley-Wiener space depending on the support of $\mathrm{d} \mu$. We have also presented constructive algorithms for practical computation of $\Phi$ and a number of examples.

Future research in this area is likely to focus on three general themes.

- Firstly, generalising the basic idea underlying this paper, 'good' approximation of a differentiation matrix in the setting of spectral methods. Are there any other separable Hilbert spaces, except for $\mathrm{L}_{2}(\mathbb{R})$, that give raise to skew-symmetric, tridiagonal, irreducible differentiation matrices? How does one obtain such orthogonal systems? The question is of great relevance insofar as $\mathrm{L}_{2}[-1,1]$, say, and $\mathrm{L}_{2}[0, \infty)$ are concerned, when the Fourier transform-based tools of this paper are no longer applicable — at least not in a straightforward manner.

Likewise, what about higher-order differentiation matrices, 'encoding' higher derivatives? In particular, approximating the second derivative with a negative semidefinite matrix is of major importance because of the ubiquity of the Laplace operator. Of course, the quindiagonal matrix $\mathcal{D}^{2}$ describes the action of second derivative in $\Phi$, and as long as $\mathcal{D}$ is skew-symmetric, $\mathcal{D}^{2}$ is negative semidefinite. However, can we find $\Phi$ with a tridiagonal, negative semidefinite, irreducible second-order differentiation matrix?

- Secondly, what are the features of orthogonal function systems $\Phi$ which act on $\mathrm{L}_{2}(\mathbb{R})$ and possess a skew-symmetric, tridiagonal, irreducible differentiation matrix $\mathcal{D}$ ? For example, where are their zeros? Brief examination of Figs. 1, 2, and 3 seems to indicate that transformed Chebyshev functions of the second kind and transformed Freud functions have an infinity of real zeros, while each Carlitz function $\varphi_{n}$ has exactly $n$ real zeros. Intriguingly, in all three cases the zeros of $\varphi_{n}$ and of $\varphi_{n+1}$ seem to interlace.

A feature which is of central importance, once we wish to harness $\Phi$ in a spectral method, is its approximation power. How well do the $\varphi_{n} \mathrm{~s}$ approximate an arbitrary $\mathrm{L}_{2}(\mathbb{R})$ function?

Many dispersive equations of quantum mechanics have solutions which can be conveniently expressed (for simplicity, in one space dimension) in terms of wave packets $\mathrm{e}^{-\alpha\left(x-x_{0}\right)} \cos \omega x$, where $\alpha>0$ and $\omega \gg 1$. How well does $\Phi$ approximate wave packets? Initial exploration indicates that there are substantial differences in using different orthogonal systems insofar as the number of terms for fixed error tolerance is concerned, once $\omega$ becomes very large and the wave packet oscillates rapidly.

- Finally, how do we implement this entire body of ideas in a practical spectral algorithm? Our ambition is an algorithm applicable to an arbitrary (in the first stage linear) time-dependent partial differential equations which is stable by design and conserves Euclidean norm whenever this norm is conserved by the original 
equations. Even disregarding the entire issue of time discretisation, practical implementation of the body of ideas in this paper requires much further research. How can we rapidly compute expansions of $L_{2}(\mathbb{R})$ functions in the basis $\Phi$ ? How do we generate such a basis in a rapid and stable manner? We expect further papers to address this issue.

Another issue is how to make the tridiagonal structure of $\mathcal{D}$ act fully to our benefit. For example, are there effective ways of approximating the product $\mathrm{e}^{c \mathcal{D}} \boldsymbol{v}$, where $c \in \mathbb{R}$ and $\boldsymbol{v} \in \ell_{2}(\mathbb{R})$, to high precision? More problems of this kind are likely to emerge once orthogonal systems, as described in this paper, are used as the kernel of a competitive spectral method.

Acknowledgements The second author is grateful to Research Foundation-Flanders (FWO) who funded him as a postdoctoral research fellow at KU Leuven during the research and writing of this paper. The authors are grateful to Peter Clarkson for discussion on Freud polynomials.

Open Access This article is distributed under the terms of the Creative Commons Attribution 4.0 International License (http://creativecommons.org/licenses/by/4.0/), which permits unrestricted use, distribution, and reproduction in any medium, provided you give appropriate credit to the original author(s) and the source, provide a link to the Creative Commons license, and indicate if changes were made.

\section{References}

1. Bader, P., Iserles, A., Kropielnicka, K. \& Singh, P. (2014), 'Effective approximation for the semiclassical Schrödinger equation', Found. Comput. Math. 14(4), 689-720.

2. Carlitz, L. (1959), 'Bernoulli and Euler numbers and orthogonal polynomials', Duke Math. J. 26, 1-15.

3. Chihara, T. S. (1978), An Introduction to Orthogonal Polynomials, Gordon and Breach Science Publishers, New York-London-Paris. Mathematics and its Applications, Vol. 13.

4. Clarkson, P. A. \& Jordaan, K. (2018), 'Properties of generalized Freud polynomials', J. Approx. Theory 225, 148-175.

5. Deift, P., Kriecherbauer, T., McLaughlin, K. T.-R., Venakides, S. \& Zhou, X. (1999), 'Strong asymptotics of orthogonal polynomials with respect to exponential weights', Comm. Pure Appl. Math. 52(12), 1491-1552.

6. Favard, J. (1935), 'Sur les polynomes de Tchebicheff"', C.R. Acad. Sci. Paris 200, 2052-2053.

7. Fokas, A. S., Its, A. R. \& Kitaev, A. V. (1992), 'The isomonodromy approach to matrix models in 2D quantum gravity', Comm. Math. Phys. 147(2), 395-430.

8. Golub, G. H. \& Welsch, J. H. (1969), 'Calculation of Gauss quadrature rules', Math. Comp. 23 (1969), 221-230; addendum, ibid. 23(106, loose microfiche suppl), A1-A10.

9. Hahn, W. (1935), 'Über die Jacobischen Polynome und zwei verwandte Polynomklassen', Math. Z. 39(1), 634-638.

10. Hairer, E. \& Iserles, A. (2016), 'Numerical stability in the presence of variable coefficients', Found. Comput. Math. 16(3), 751-777.

11. Hairer, E. \& Iserles, A. (2017), 'Banded, stable, skew-symmetric differentiation matrices of high order', IMA J. Numer. Anal. 37(3), 1087-1103.

12. Iserles, A. (2014), 'On skew-symmetric differentiation matrices', IMA J. Numer. Anal. 34(2), 435-451.

13. Iserles, A. (2016), 'The joy and pain of skew symmetry', Found. Comput. Math. 16(6), 1607-1630.

14. Konoplev, V. P. (1961), 'Polynomials orthogonal with respect to weight functions which are zero or infinite at isolated points of the interval of orthogonality', Dokl. Akad. Nauk SSSR 141, 781-784.

15. Levin, E. \& Lubinsky, D. S. (2001), Orthogonal Polynomials for Exponential Weights, Vol. 4 of CMS Books in Mathematics/Ouvrages de Mathématiques de la SMC, Springer-Verlag, New York.

16. Magnus, A. P. (1995), 'Painlevé-type differential equations for the recurrence coefficients of semiclassical orthogonal polynomials', J. Comput. Appl. Math. 57(1-2), 215-237.

17. Olver, F. W. J., Lozier, D. W., Boisvert, R. F. \& Clark, C. W., eds (2010), NIST Handbook of Mathematical Functions, U.S. Department of Commerce, National Institute of Standards and Technology,

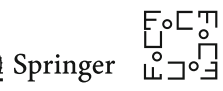


Washington, DC; Cambridge University Press, Cambridge. With 1 CD-ROM (Windows, Macintosh and UNIX).

18. Rainville, E. D. (1960), Special Functions, The Macmillan Co., New York.

19. Shohat, J. (1939), 'A differential equation for orthogonal polynomials', Duke Math. J. 5(2), 401-417.

20. Stein, E. M. \& Weiss, G. (1971), Introduction to Fourier Analysis on Euclidean Spaces, Princeton University Press, Princeton, N.J. Princeton Mathematical Series, No. 32.

21. Szegő, G. (1918), 'Ein Beitrag zur Theorie der Polynome von Laguerre und Jacobi', Math. Z. 1(4), 341356.

22. Szegó, G. (1975), Orthogonal Polynomials, fourth edn, American Mathematical Society, Providence, R.I. American Mathematical Society, Colloquium Publications, Vol. XXIII.

23. Van Assche, W. (2018), Orthogonal Polynomials and Painlevé Equations, Vol. 27 of Australian Mathematical Society Lecture Series, Cambridge University Press, Cambridge.

Publisher's Note Springer Nature remains neutral with regard to jurisdictional claims in published maps and institutional affiliations. 